\title{
Newly-identified receptors for peptide histidine-isoleucine and GHRH-like peptide in zebrafish help to elucidate the mammalian secretin superfamily
}

\author{
Sheng Wu, Graeme J Roch, Laura A Cervini ${ }^{1}$, Jean E Rivier ${ }^{1}$ and Nancy M Sherwood \\ Department of Biology, University of Victoria, Victoria BC, Canada V8W 3N5 \\ ${ }^{1}$ Peptide Biology Laboratory, The Salk Institute, 10010 N. Torrey Pines Road, La Jolla, California 92037, USA \\ (Correspondence should be addressed to N M Sherwood; Email: nsherwoo@ uvic.ca)
}

\begin{abstract}
A group of ten hormones in humans are structurally related and known as the secretin superfamily. These hormones bind to G-protein-coupled receptors that activate the cAMP pathway and are clustered as the secretin or B family. We used an evolutionary approach with zebrafish as a model to understand why some of these hormones, such as peptide histidinemethionine (PHM) and pituitary adenylate cyclase-activating polypeptide (PACAP)-related peptide (PRP) in humans lack a receptor. We used molecular techniques to clone two full-length receptor cDNAs in zebrafish, which were analyzed for amino acid sequence and ligand-binding motifs, phylogenetic position, synteny, tissue expression, functional response, and signaling pathway. Evidence is provided that the two cDNAs encoded the peptide histidine-isoleucine (PHI) receptor and PRP receptor, which is known as GHRH-like peptide (GHRH-LP) receptor in non-mammals. Further, we cloned a zebrafish cDNA encoding the peptides $\mathrm{PHI}$ and vasoactive intestinal peptide (VIP). The PHIR had been previously labeled as one type of a VIP-PACAP (VPAC2R) shared receptor based only on sequence data. The PHIR cDNA, transfected into COS7 cells, responded to zebrafish $\mathrm{PHI}$ in a sensitive and dose-dependent manner $\left(E \mathrm{EC}_{50}=1 \cdot 8 \times\right.$ $10^{-9} \mathrm{M}$ ) but not to PACAP and VIP. The GHRH-LP receptor responded to both zebrafish GHRH-LP1 and GHRH with a 3.5-fold greater response to the former. For comparison, two zebrafish receptors (PAC1R and VPAC1R) and two human receptors (VPAC2R and GHRHR) were tested with human and/or zebrafish peptides. Unexpectedly, zebrafish VIP activated its PAC1R suggesting that in evolution, PAC1R is not always a specific receptor for PACAP. We conclude that zebrafish, like goldfish, have a specific receptor for $\mathrm{PHI}$ and GHRH-LP. Our evidence that zebrafish PHI is more potent than human PHM in activating the human VPAC2R $\left(E C_{50}=7 \cdot 4 \times 10^{-9} \mathrm{M}\right)$ supports our suggestion that the VPAC2R and PHIR shared a common ancestral receptor.
\end{abstract}

Journal of Molecular Endocrinology (2008) 41, 343-366

\section{Introduction}

In humans, the secretin superfamily has ten hormones and at least nine receptors, all of which are listed in Table 1 with abbreviations and gene names. To date, one of the hormones lacks a specific (high-affinity) receptor and several other hormones share receptors. Only three of the ten hormones are encoded in a single gene, whereas the other hormones are encoded as two or three hormones within a gene. One human gene encodes both pituitary adenylate cyclase-activating polypeptide (PACAP) and PACAP-related peptide (PRP). Another human gene encodes both vasoactive intestinal polypeptide (VIP) and peptide histidinemethionine (PHM), whereas a third gene encodes glucagon and glucagon-like peptides 1 and 2. The remaining three genes each encode only a single hormone: growth hormone-releasing hormone (GHRH), secretin (SCT), or glucose-dependent insulinotropic polypeptide (GIP). The nine receptors that bind these hormones are also structurally related as seven transmembrane receptors, which are grouped within the type B (or type 2) family of G-proteincoupled receptors (Sherwood et al. 2000, Ulloa-Aguirre \& Conn 2000, Cardoso et al. 2005) and named by the International Union of Pharmacology (Foord et al. 2005). An understanding of the evolutionary history of the secretin superfamily of hormones and receptors may help to explain the gene organization, receptor binding, and function of this group of hormones.

We selected zebrafish as a comparison with humans to probe several critical questions: 1) why do peptide histidine-isoleucine (PHI; non-human) and PHM (human) lack a specific receptor with high-affinity binding, 2) why does VIP lack a specific receptor, 3) why are there two shared receptors (VPAC1R and VPAC2R) that are activated equally by both PACAP and VIP, 4) why does PRP not have a receptor, and 5) is there a full complement of secretin superfamily hormones and receptors in zebrafish? An examination of these 
Table 1 The secretin superfamily of hormones and receptors in zebrafish and humans

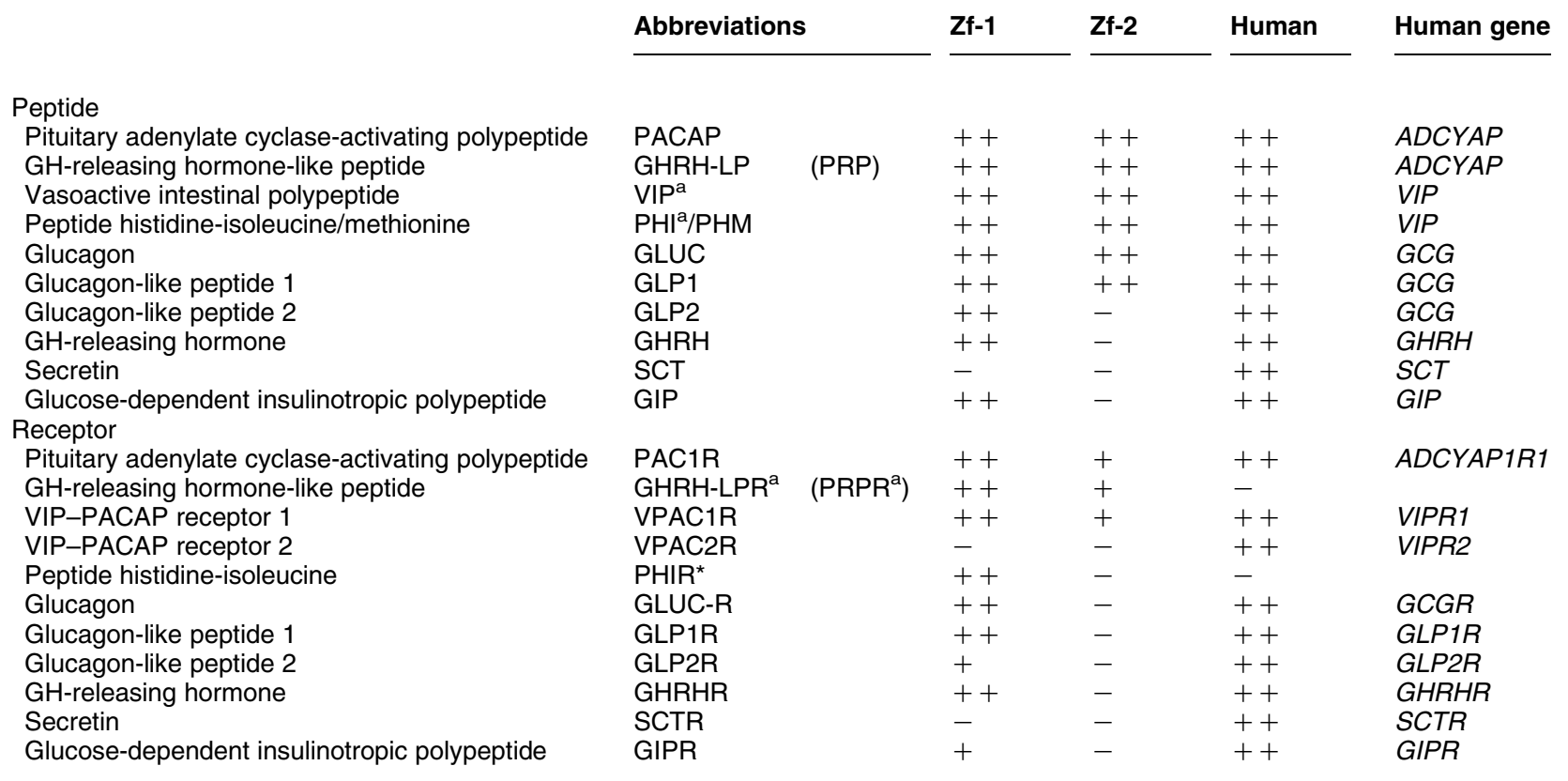

${ }^{++}$mRNA transcribed; ${ }^{+}$peptide or receptor identified in genome but not isolated; Alternate names include GHRH-LP in non-mammals but PACAP-related peptide or PRP in mammals; PHI in most vertebrates is PHM in humans; references and structures are at http://www.ncbi.nlm.nih.gov.

aldentified in zebrafish in the present paper.

hormones and receptors in zebrafish is of considerable interest, as this fish is a well-known model for developmental and endocrine studies (McGonnell \& Fowkes 2006).

Of the ten hormones in humans, PACAP is the most conserved member. The two biologically active forms, PACAP $_{27}$ and PACAP $_{38}$, are identical as the latter is a C-terminal extension of $\mathrm{PACAP}_{27}$ (Miyata et al. 1989, 1990). Zebrafish also have PACAP $_{27}$ and PACAP $_{38}$, but the gene encoding the peptides has been duplicated producing two forms of each peptide (Fradinger \& Sherwood 2000, Wang et al. 2003). PACAP has a wide range of functions as a neuromodulator, neurotropin, smooth muscle relaxant, and a releasor of pituitary hormones (Sherwood et al. 2000, Chow et al. 2003). In fish, PACAP additionally can release growth hormone (Parker et al. 1997, Wong et al. 1998). The receptors that activate these physiological actions are PAC1R, which preferentially binds PACAP with high affinity compared with VIP, and VPAC1R that shows similar potency with PACAP or VIP (Laburthe et al. 2003). Both PAC1R and VPAC1R are identified in humans and zebrafish, but humans also have a VPAC2R that is related in structure and function to VPAC1R. Fish have receptors that resemble VPAC2Rs in structure, but they have not been tested for binding to any peptides.

Although PRP and PACAP share the same precursor, the function of PRP in mammals is unknown. In human, PRP is $52 \%$ identical in amino acids to $\mathrm{GHRH}_{27}$, whereas in zebrafish one of the two PRP peptides, known as GHRH-like peptide1 (GHRH-LP1) has a higher identity $(59 \%)$ with the zebrafish true $\mathrm{GHRH}_{27}$. Also, release of $\mathrm{GH}$ has been observed in some fish experiments but not in others after application of GHRH-LP in vivo or in vitro (see Tam et al. 2007 for review). A PRP receptor has not been identified for any mammal but a full-length sequence (PRPR or alternatively GHRH-LPR) in goldfish (Chan et al. 1998) and partial sequence (transmembrane domains 5-7) in zebrafish (Fradinger et al. 2005) have been reported.

Another two neuropeptides, PHM and VIP, are listed in Table 1; they are cleaved from a single precursor resulting in $\mathrm{PHM}_{27}$ and $\mathrm{VIP}_{28}$ in humans with $44 \%$ amino acid identity. VIP is a potent peptide that mediates smooth muscle relaxation, stimulates endocrine and exocrine secretion, regulates circadian rhythms and neuromodulates, whereas PHM is thought to be a less potent version of VIP that uses the same receptors, VPAC1R and VPAC2R. The name of PHM in human becomes PHI in other vertebrates, as the C-terminal peptide is isoleucine (I) rather than methionine (M). In zebrafish, a PHI/VIP cDNA has not been cloned. The receptors for VIP are shared with PACAP as noted earlier. However, a receptor for PHM/ PHI has not been identified for any mammal and only one PHIR (goldfish) has been characterized to date (Tse et al. 2002). 
Mammalian GHRHs act on the pituitary to release GH. A 'true' GHRH cDNA was identified only in 2007 for chicken (Wang et al. 2007), goldfish, and zebrafish (Lee et al. 2007), and shown to be a homolog with the mammalian form. Relevant here, the zebrafish and goldfish GHRH receptors have been shown to be homologs of the mammalian receptor using synteny and response to fish true GHRH in vivo and in vitro (Lee et al. 2007).

In the present experiments, our aim was to use zebrafish to identify a PHIR and determine which signaling pathway is coupled to this receptor. To test the biological activity of the PHIR, we cloned the PHI/VIP cDNA precursor and synthesized the two peptides. The zebrafish peptides of PHI and VIP along with zebrafish PACAP1 and 2 were used to test the signaling path of the zebrafish PHIR. To further expand our understanding of the evolution of the secretin superfamily, we also cloned and expressed GHRH-LP receptor and characterized its signaling properties. Human and zebrafish peptides were tested also on human VPAC2R and GHRHR. Our hypothesis is that the evolution of the secretin superfamilies of hormones and receptors in vertebrates has resulted in the loss or alteration of certain receptors in mammals with subsequent changes in ligand binding. Phylogenetic analysis and synteny maps were used to evaluate the evolution of the receptors.

\section{Materials and methods}

\section{Animals}

Wild-type zebrafish (Danio rerio) were purchased from a local pet store and maintained in our laboratory with a $14 \mathrm{~h}$ light: $10 \mathrm{~h}$ darkness cycle at $28.5^{\circ} \mathrm{C}$. Procedures were approved by the Animal Care Committee at the University of Victoria. The tissues of adult zebrafish were dissected and frozen in liquid nitrogen.

\section{Peptides}

The sequences of seven zebrafish peptides used to test receptor activity are shown in Table 2 . All peptides were synthesized at the Salk Institute (La Jolla, CA, USA) using a CS-biopeptide synthesizer (model CS536, CS Bio Co. Inc., San Carlos, CA, USA) on a methyl benzhydrylamine resin using Boc-strategy. The peptides were cleaved with hydrofluoric acid, concomitantly deprotected, and then purified as described in Adams et al. (2003). The purity of the peptides was characterized by capillary zone electrophoresis performed on a Beckman P/ACE System 2050 connected to a ChromJet integrator (Spectra Physics, San Jose, CA, USA). Matrix-assisted laser desorption/ionization time-of-flight mass spectrometry of the peptides was measured on an ABI Perseptive DE-STR instrument (PE Applied Biosystems, Foster City, CA, USA). In addition, three human peptides were tested: $\mathrm{PHM}_{27}$ and $\mathrm{VIP}_{28}$ (Bachem, Torrance, CA, USA), and $\mathrm{GHRH}_{44}$ (Salk Institute).

\section{Total RNA isolation and cDNA synthesis}

Total RNA was extracted from 11 tissues of adult zebrafish: brain, eye, heart, testis, ovary, spleen, kidney, gill, skin, gut, and swimbladder according to the manufacturer's protocol (RNeasy Mini Kit, Qiagen Inc.) The concentration of total RNA was measured and $900 \mathrm{ng}$ total RNA from each sample was reverse transcribed with Oligo (dT) and Superscript II (Invitrogen) according to the manufacturer's instructions.

\section{RACE reactions for zebrafish phir, ghrh-Ipr, and vip (PHI/VIP)}

Total RNA (500-600 ng) from adult zebrafish brains was used for the synthesis of cDNA with the First Choice RLMRACE kit (Ambion) following the manufacturer's instructions. The $5^{\prime}$ and $3^{\prime}$ RACE primers for phir were designed from a published partial sequence for vipr2 (VPAC2R) (Wang et al. 2003) and ghrh-lpr (Fradinger et al. 2005). A partial predicted zebrafish VIP peptide sequence was found by performing a BLAST search with VIP of rat/ mouse, guinea pig, chick, frog, trout/bowfin, cod, dogfish in the Vega Multi Blast View program (http:/ / vega.sanger. ac.uk/Multi/blastview? species $=$ D._rerio); this partial

Table 2 Amino acid sequences of the zebrafish peptides tested in the receptor activation assay

\section{Sequence}

\section{Peptide}

PACAP1 $_{27}$

$\mathrm{PACAP}_{27}$

$\mathrm{VIP}_{28}$

$\mathrm{PHI}_{27}$

GHRH-LP1 $1_{28}$

GHRH-LP2 29

$\mathrm{GHRH}_{27}$

\begin{abstract}
HSDGVFTDSYSRYRKQMAVKKYLATVL- $\mathrm{NH}_{2}$ HSDGIFTDIYSRYRKQMAVKKYLAAVL- $\mathrm{NH}_{2}$ HSDAIFTDNYSRFRKQMAVKKYLNSVLT-NH HADGIFTSGYSKLLGQLSARRYLESLI-NH HADGMFNKAYRKALGQLSARKYLHTLMA HADGLLDRALRDILVQLSARKYLHSLMAV HADAIFTNSYRKVLGQISARKFLQTVM-NH $\mathrm{N}_{2}$
\end{abstract}

\section{References}

Fradinger et al. (2000)
Wang et al. (2003)
(Present results)
(Present results)
Fradinger et al. (2000)
Wang et al. (2003)
Lee et al. (2007) and Wang et al.
(2007)


predicted zebrafish VIP peptide was identical to trout VIP. Primers for $3^{\prime}$ RACE of vip (PHI/VIP) were designed to this predicted nucleotide sequence. Later, $5^{\prime}$ RACE primers were designed from our $3^{\prime}$ RACE PCR sequence. RACE-PCRs were carried out for 35 cycles at $94^{\circ} \mathrm{C}$ for $30 \mathrm{~s}$, $55{ }^{\circ} \mathrm{C}$ annealing temperature for $30 \mathrm{~s}$, and $72{ }^{\circ} \mathrm{C}$ for $1 \mathrm{~min}$ with a 7 -min extension at $72^{\circ} \mathrm{C}$ on the last cycle. All primers are listed in Table 3. The PCR products were separated by electrophoresis on a $1.5 \%$ agarose gel and stained with ethidium bromide. Then, PCR products were selected and isolated with QIAquick Gel Extraction Kit (Qiagen), and ligated into PGEM-T vector (Promega Corp.)

\section{Data mining, phylogenetic analysis, and synteny comparisons}

The NCBI database was used to search for PAC1R, VPAC1R, VPAC2R, GHRHR and GHRH-LPR in human,

Table 3 Primers used to amplify cDNA in zebrafish

Primers
RACE
phir
$\mathrm{R}^{\mathrm{a}} 274 \mathrm{a}$
R267b
$\mathrm{F}^{\mathrm{b}} 3 \mathrm{~b}$
F191
ghrh-Ipr
R-615
R-190
vip (PHI/VIP)
R706
R584
F260
Clones for functional study
phir
F1
R2096
F172Nhel
R1551Xbal
ghrh-Ipr
F5
R1675
F120Nhel
R1436Xhol
Tissue expression primers
phir
F429
R853
ghrh-Ipr
F79
R701
ghrhr
F30
R625
vip (PHI/VIP)
F41
R596
vipr1 (VPAC1R)
F66
R537
adcyap1r1 (PAC1R)
F1123
R1613
F-actin1
R1033

The enzyme restriction sites are underlined. ${ }^{\mathrm{a}} \mathrm{F}$, forward primer.

${ }^{\mathrm{b}} \mathrm{R}$, reverse primer.
Sequence $\left(5^{\prime}-3^{\prime}\right)$

Target

CAGGTTCAGATGGATGTAGT

AGATGGATGTAGTTCCTGGTG

GTCCTGCTGCCCTCAAAA

CTCATCGCCCTCATCACC

TCCAGAACGCCAGTCGTCATCAA

CCCCGACTCCAGGACTTTTC

AATAAAGATTTCCAATGCCCGACCC

TCATCGTCCAATCAGCAGTC

GTAGTGATGATTTGATGGAAGACC

$5^{\prime}$ CDNA

$5^{\prime}$ CDNA

$3^{\prime}$ CDNA

$3^{\prime}$ CDNA

$5^{\prime}$ cDNA

$5^{\prime}$ CDNA

$5^{\prime}$ CDNA

$5^{\prime}$ CDNA

$3^{\prime}$ CDNA

cDNA

AAAGTACTGCAAACCGCACATGAT

CTTACAATCACCGCTTCACA

TGCTAGCGCCACCATGGACATGAGCCTCGTCAACT

ATTCTAGAAGGGCAGTGAAGAGCGAGCG

AAGTGAATCTGACTGTGCG

ATCATATATATTTTTAAAATCATTCTTATTTCC

TTGCTAGCGCCACCATGGCGGTTGTACATCTGTGCT

TTCTCGAGTACAATCCAGTGGCGAGTTCA

CDNA
CAGAGATGGGATCATTAGCAG

GTAAGTACAATCCTTCAACCAG

GTCGATGAGAAAGAGCGGCTGT

GAAGAAACATCCTCAAGACAATACTG

CCGTGTGCGCGTGGACTTTT

CAGGCCACAGTAGACAGAGAGCAG

CAGGTGTCCACGGCTGTGTG

AGGGCTCCATCGAAACGCAG

AACTTGCTATGTTTTACTCGTCTG

ATCGTCAACAGTGCCATTCA

GCCCTGTGGTGGCATCAATC

TCAGGTTGGCAGGTTGAGGT

ATTGCCGCACTGGTTGTT CTCCGATCCAGACAGAGTATTTT 
chicken, frog, goldfish, fugu, and zebrafish genomes. The amino acid sequence of the open reading frame of zebrafish PHI receptor with its seven transmembrane domains was used separately to search for homologous receptors in the same genomes as above and in those of medaka (Oryzias latipes), stickleback (Gasterosteus aculeatus), Tetraodon nigroviridis, and salmonids (Salmonidae family) not only in NCBI website (www.ncbi.nlm.nih.gov) but also in Ensembl (www.ensembl.org) and the Salmon Genome Project database (www.salmongenome.no). The deduced amino acid sequences were aligned using the ClustalW alignment program (Thompson et al. 1994) with BLOSUM62. A neighborjoining (NJ) phylogenetic tree was generated based on the ClustalW alignment of the amino acids from transmembrane domains 1-7 by MEGA3.0 (Kumar et al. 2004) to produce the tree using JTT (Jones-TaylorThornton) substitution. In addition, a maximumlikelihood (ML) tree was generated with 100 bootstraps and the JTT substitution using PHYML (Guindon \& Gascuel 2003) and its online web server (http://atgc. lirmm.fr/phyml/menu.html; Guindon et al. 2005).

The GenBank accession numbers for the receptors isolated here are zebrafish PHIR (EU150381) and zebrafish GHRH-LPR (EU150382). For other sequences, the numbers are: human (Homo sapiens) GHRHR (NP000814); chicken GHRHR (ABB84385); goldfish GHRHR (ABJ55978); zebrafish GHRHR (ABJ55981); chicken PRP receptor (PRPR; CAC82589); goldfish GHRH-LPR (AAC15698); fugu GHRH-LPR (CAC82589); human VPAC2R (P41587); chicken VPAC2R (NP_001014970); fugu VPAC2RA (AJ408877); fugu VPAC2RB (AJ296143); trout (AY706217), human VPAC1R (P32241); marsh frog VPAC1R (Q9YHC6); goldfish VPAC1R (AAB05459); zebrafish VPAC1R (NP_001013371.1 and for the second receptor ENSDARP00000046126); fugu VPAC1RA (CAC82588); fugu VPAC1RB (CAC82587); human PAC1R (P41586); goldfish PAC1R (O73769); zebrafish PAC1R-short (AAW65134.1 and for the second receptor XP682980); fugu PAC1RA (CAD35690); and fugu PAC1RB (CAD33842). The Ensembl accession numbers are: medaka VPAC2RA (ENSORLP00000023740), medaka VPAC2RB (ENSORLP0000007394), Tetraodon VPAC1R (GSTENP00016553001), stickleback VPAC2RA (ENSGACP0000002397), and stickleback VPAC2RB (ENSGACP00000023187).

Ensembl also lists two accession numbers for zebrafish VPAC2R, one of which (ENSDARG00000012353) is the full-length (441 amino acids) receptor that we call the PHIR and the other (ENSDARG00000067568) is a truncated (318 amino acids) receptor; the shorter receptor lacks an extracellular N-terminal domain and its 318 amino acids are $100 \%$ identical to the longer receptor. The truncated receptor is not considered further. The duplicate receptor for zebrafish VPAC1R was not included in the ML or NJ trees as the receptor had low sequence similarity in TMD 3-6, probably due to inaccurate exon predictions. However, we have included in the ML tree a second zebrafish GHRH-LPR (ENSDARP00000070262), which lacks 100 amino acids in the N-terminal domain, because the remaining amino acids include all 7 TM regions and are distinct from the first form of the receptor; the truncated receptor has not been cloned to date but the genomic sequence shows a 26 amino acid insertion compared with other GHRH-LPRs (Fig. 2). The zebrafish glucagon receptor (GLUCR) (XM_685886) was used as the outgroup for tree construction. Goldfish PHIR was reported earlier but does not have an accession number (Tse et al. 2002). Two receptors with accession numbers are not considered further as second forms in our study, as they are almost identical (99-99.5\%) to ones listed in Fig. 1 or 2; one of these receptors is a second form of fugu GHRH-LPR (ENSTRUP00000032622) and the other is a second form of trout VPAC2R (CU069615), which is only a fragment (residues 255-440) of the full-length receptor.

To examine the syntenic arrangement of genes surrounding the PHI gene and VPAC2R gene, a sequence similarity approach was used. All data were obtained from the Ensembl database. The neighboring genes of the two receptors were used to search for orthologs in zebrafish, medaka, chicken, and human genomes using the MultiContigView and then selecting 'View alongside' and the species of interest. Also, the same genes were searched in Ensembl Blast View and NCBI database to avoid missing any data. The medaka VPAC2R genes were not labeled A or B in Ensembl so we assigned A to the receptor that clustered with other fish VPAC2Rs in the ML tree (based on comparison of the seven transmembrane regions). However, medaka VPAC2RB was closer to zebrafish PHIR in a comparison of total length (Table 4). Therefore, we used the medaka $\mathrm{B}$ receptor for synteny comparisons; also the gene neighbors matched those of the zebrafish receptor and the medaka $A$ receptor could not be compared, as it was on the end of a scaffold.

\section{Tissue distribution of zebrafish mRNA: phir, adcyap1r1 (PAC1R), vipr1 (VPAC1R), ghrh-Ipr, vip (PHI/VIP)}

Tissue-specific distribution of each mRNA was performed by RT-PCR on 11 tissues of zebrafish. Primers for the adcyap1r1-short isoform, viprl, and phir were designed according to the NCBI Gene Bank database (accession No.: AAW65134.1; NP_001013371.1; NP_571854). ghrh-lpr and vip primers were designed according to our $5^{\prime}$ and $3^{\prime}$ RACE sequences. A control reaction was prepared using $1 \mu \mathrm{l}$ of cDNA amplified with zebrafish actin primers. All PCRs were carried out on $1 \mu \mathrm{l} \mathrm{cDNA}$ for 35 cycles at different annealing temperatures: $58^{\circ} \mathrm{C}$ 
Table 4 Percentage of amino acid identity of aligned zebrafish peptide histidine-isoleucine (PHI) receptor with others in the same gene family

1 Zebrafish PHIR

2. Goldfish PHIR

3. Fugu VPAC2RA ${ }^{a}$

4. Fugu VPAC2RB ${ }^{a}$

5. Trout VPAC2R

6. Tetraodon VPAC2R ${ }^{\mathrm{a}}$

7. Stickleback VPAC2RA

8. Stickleback VPAC2RB ${ }^{a}$

9. Medaka VPAC2RA ${ }^{a}$

10. Medaka VPAC2RB ${ }^{a}$

11. Chicken VPAC2R

12. Human VPAC2R

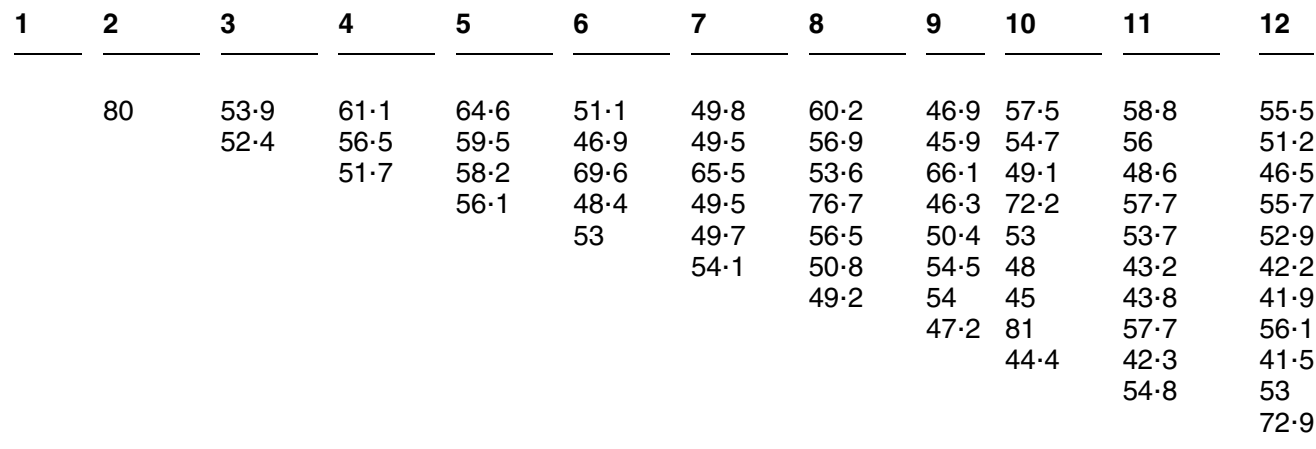

Amino acid identity was determined by using the ClustalW alignment program with BLOSUM62.

${ }^{a}$ Means receptor amino acid sequence is partial as shown in Fig. 1.

(bactin 1), $60{ }^{\circ} \mathrm{C}$ (adcyap1r1, phir), $62{ }^{\circ} \mathrm{C}$ (vip, viprl, ghrh-lpr, $g h r h r)$. All PCR products spanned at least two exons as a control check for potential genomic contamination. PCR products of the expected size were sequenced and confirmed.

\section{Transfection and expression of zebrafish PHIR, PAC1R-short, VPAC1R, GHRH-LPR and human VPAC2R and GHRHR}

Constructs of cDNA full-length coding sequences for receptors PAC1R-short and VPAC1R were a gift from Javier Tello as previously reported (Fradinger et al. 2005). The cDNAs for PHIR and GHRH-LPR containing full-length open reading frames with flanking restriction enzymes sites and a Kozak sequence were subcloned into pcDNA3.1 ( - ) (Invitrogen). Two rounds of PCRs were conducted with platinum Taq polymerase high fidelity (Invitrogen) as outlined by the manufacturer. Primers are listed in Table 3. Human vasoactive intestinal peptide receptor 2 (VPAC2R) and GHRHR cDNA clones were obtained from University of Missouri-Rolla (UMR) cDNA Resource Center (Clone ID: VPAC2R00000, GHRHR00000; GB Acc. No.: NM_003382, AY557192). All six receptors were separately transfected into COS7 cells (American Type Culture Collection, Manassas, VA, USA) as described (Tello et al. 2005) with a few modifications: 1) cells were plated at a density of 60000 cells/well, 2) medium was replaced after $14 \mathrm{~h}$ of transfection with fresh VP-SFM (Invitrogen) for later cAMP assay or replaced with labeling medium (Medium 199, Invitrogen) plus $0.3 \%$ bovine albumin (SigmaAldrich) for the inositol phosphate (IP) assay, and 3) labeling medium with isobutylmethylxanthine (IBMX) and $10 \mathrm{mM} \mathrm{LiCl}$ was used to incubate cells during ligand stimulation. The cAMP assay was done with an Amersham cAMP enzyme immunoassay Biotrak system
(GE Healthcare, Little Chalfont Buckinghamshire, UK). In the total IP accumulation assay, quantitation was performed with cell extracts by the multi-well filtration method (Chengalvala et al. 1999).

\section{Data analysis}

All IP and cAMP samples were measured in triplicate within each assay and each experiment was independently repeated at least thrice. Data analysis was performed using PRISM3 software (GraphPad Software Inc., San Diego, CA, USA) with nonlinear regression (curve fit) and sigmoidal dose-response curves. The $\mathrm{EC}_{50}$ values (dose of peptide stimulating half-maximal IP or cAMP response) of each peptide were calculated from the means of at least three independent experiments. The data were analyzed by one-way ANOVA followed by Tukey's multiple comparison test. $P<0.05$ was considered as statistically significant.

\section{Results}

\section{Isolation and structural analysis of zebrafish PHIR, GHRH-LPR, and PHI/VIP cDNAs}

Full-length cDNAs obtained with the use of total RNA from zebrafish brains and RACE reactions were sequenced in both the forward and reverse directions. The zebrafish PHIR cDNA was $2088 \mathrm{bp}$ in length (GenBank accession No. EU150381) with an open reading frame of $1323 \mathrm{bp}$ (from 169-1491) encoding a 441 amino acid protein (Fig. 1A and B). A Kyte-Doolittle hydrophobicity plot (data not shown) of the deduced protein revealed that it has seven transmembrane domains. Comparison of the zebrafish PHIR deduced amino acid sequence of the full receptor revealed that its 
A

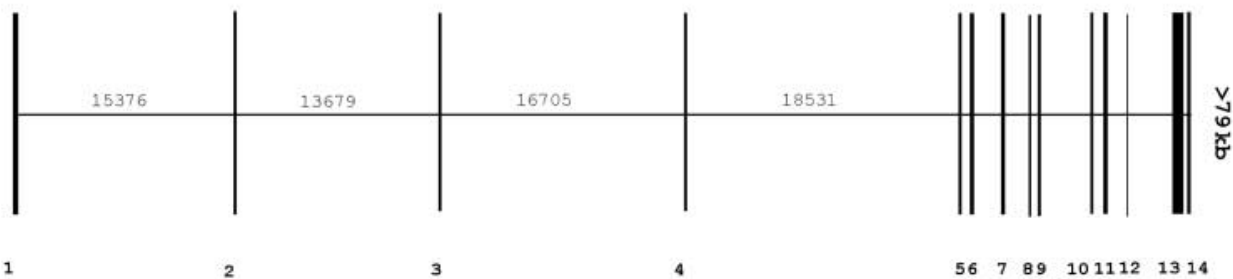

B

Zebrafish PHIR Goldfish PHIR

Fugu VPAC2RA

Fugu VPAC2RB

Trout VPAC2R

Tetraodon VPAC2R

Stickleback VPAC2RA

Stickleback VPAC2RB

Medaka VPAC2RA

Medaka VPAC2RB

Chicken VPAC2R

Human VPAC2R

Clustal consensus

Zebrafish PHIR Goldfish PHIR Fugu VPAC2RA

Fugu VPAC2RB

Trout VPAC2R

Tetraodon VPAC2R

Stickleback VPAC2RA

Stickleback VPAC2RB

Medaka VPAC2RA

Medaka VPAC2RB

Chicken VPAC2R

Human VPAC2R

Clustal consensus
Signal peptide

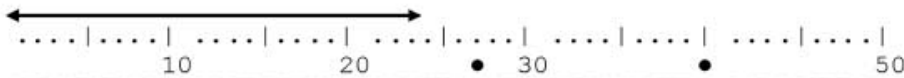
MNMSLVNCLV LLIVWHAHSV NGRHPNCQFL YELQMAEKEC LGDLADEPLL MKMNLVNCLA LLLVWHAHAV NARHANCHFL YELQMAEKEC LDMLADELPP -------- --------V HGKFPHCQFY WEMQRARRDC QIQLQRE--T -------- ------- EGRHPNCNFL LEVERDHAEC LRRLR-EEKE MERSRVGLLF LALCLLRVKV SGRNPTCHLL WELQRAELVC HYELKKQSME -------- -------- -----CQFH WEMERARREC QIQLQRE--S ----------V NGKLTHCQFQ WEMQRARREC DTLLQRSNVC

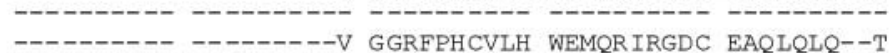

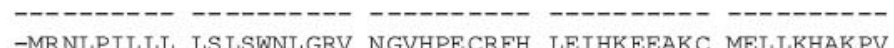
-MRTLLPPAL LT-CWLLAPV NSIHPECRFH LEIQEEETKC AELLR-SQTE

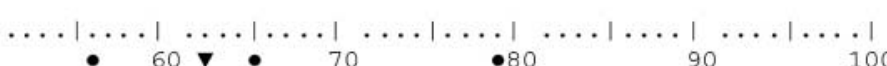
HTEG-CRGMW DNFSCWHHAD VGKVVVNNCP AALKNLETRD G-------HTQG-CRGIW DNFSCWHHAD IGEVVDNTCP VALKNLFTRD G--------SAHTGCRGEW DNVSCWQSAA VGEVTTLPCP SPLLHLFGKK G--------ATSS-BKGVW DSIACWERAE VGEIVTIPCP RVLKTVFGRN G--------PAG--CGGLW DNISCWAPAA VGEVVTLSCP PALTHLFGRQ G--------HANAGCRGEW DNVLCWQSAA VGEVTTLPCP SALQHLFGRN GPYRVLVSEN VCGSGCLGEW DNVSCCQRAA VEEVLTLPGP PLLVSLFGNN G-------$---A-C R G T W$ DSIACWERTA VGQTVTIPCP RVLTTVFGRN G--------AAAAGCPGEW NNVSCWPNAA VGEVLTLPCP SPFLLFFEKK D--------------KGTW ISIACWEHTG LGETVTIPCP RALKTIFGRN G--------DYKG-CVGVW DNITCWRPAK IGETVTVPCP KIFSLVSGKP G--------KHKA-CSGVW DNITCWRPAN VGETVTVPCP KVFSNFYSKA G-------* $: .{ }^{*}:$ : : $\quad$ * $\quad$ : . .
Zebrafish PHIR Goldfish PHIR Fugu VPAC2RA Fugu VPAC2RB Trout VPAC2R Tetraodon VPAC2R stickleback VPAC2RA Stickleback VPAC2RB Medaka VPAC2RA Medaka VPAC2RB Chicken VPAC2R Human VPAC2R Clustal consensus
Figure 1 (continued) 
Zebrafish PHIR Goldfish PHIR Fugu VPAC2RA Fugu VPAC2RB Trout VPAC2R Tetraodon VPAC2R stickleback VPAC2RA Stickleback VPAC2RB Medaka VPAC2RA Medaka VPAC2RB Chicken VPAC2R Human VPAC2R Clustal consensus

\begin{abstract}
Zebrafish PHIR Goldfish PHIR Fugu VPAC2RA Fugu VPAC2RB Trout VPAC2R Tetraodon VPAC2R stickleback VPAC2RA Stickleback VPAC2RB Medaka VPAC2RA Medaka VPAC2RB Chicken VPAC2R Human VPAC2R
\end{abstract} Clustal consensus

Zebrafish PHIR
Goldfish PHIR
Fugu VPAC2RA
Fugu VPAC2RB
Trout VPAC2R
Tetraodon VPAC2R
Stickleback VPAC2RA
Stickleback VPAC2RB
Medaka VPAC2RA
Medaka VPAC2RB
Chicken VPAC2R
Human VPAC2R
Clustal consensus

Zebrafish PHIR
Goldfish PHIR
Fugu VPAC2RA
Fugu VPAC2RB
Trout VPAC2R
Tetraodon VPAC2R
Stickleback VPAC2RA
Stickleback VPAC2RB
Medaka VPAC2RA
Medaka VPAC2RB
Chicken VPAC2R
Human VPAC2R
Clustal consensus

Figure 1 (continued)

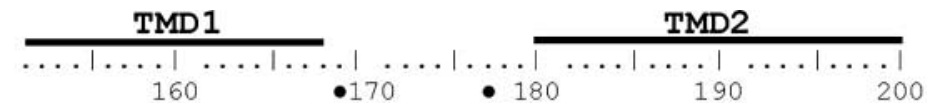
TLGHSLSLIA LITGSTILCL FR-KLHCTRN YIHLNLFFSF ILRAIAVLVK TLGHSLSLIA LITGSTILCL FR-KLHCTRN YIHLNLFFSF ILRAIAVLVK TLGHSLSLVT LLTSTFIMCF FR-RLHCTRN YIHLNLFVSF MLRAVAVLAK TLGHSLSLIA LITGSAVLCL FR-KLHCTRN YIHLNLFLSF ILRAVAVLAK TLGHSLSLIA LITSTLILCL FR-RLHCTRN YIHVNLIVSF ILRAVAVLVK TLGYSLSLVA LLASTFIVCF FR-RLHCTRN YIHLNLFVSF MLRAVAVLAK TLGHSLTFIT LLTSTVIICL YR-KLHCTRN YVHLNLFLSF MLRAAAVLSK TLGHILSLIA LTTGSAILCL FR-KLHCTRN YIHLNLFFSF VLRAVAVLVK -VSNTSTLSE FLEMSFKSCF LK-RLHCTRN YIHVNLFVSE MLRAVAVLAK TLGHSLSLIA LITGSAILCL FR-KLHCTRN YIHLNLFVSF ILRAVAVLVK TLGHSVSLIA LTTGSIILCL FR-KLHCTRN YIHLNLFLSF ILRAISVLVK TLGYSVSLMS LATGSIILCL FR-KLHCTRN YIHLNLFLSF ILRAISVLVK

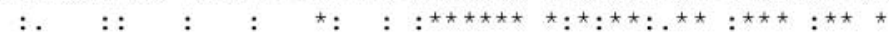
$\left.\left.\cdots|\cdots|\right|_{210} \cdots|\cdots|_{220} \cdots|\cdots|\right|_{230} \cdots \mid \frac{m_{240} \cdots|\cdots|}{250}$ DAILFS-HEN GECTVQP-SL M--------- --GCKVSLVI LNYFIMANFY DAILFS-HGN VECSKHP-SL VNESIHPEYL QIGCKASLII FHYFIIANFY DTLLFSDDET TDCSTQP-SL V------- --GCKAILVF FNYFVMANFF DDI LFN--RT SQCSNQP-SL AG---- --- ---CKTSLVF FQYFIMANFF DSVIFSHNEN TDCSTQP-SL VG-------- ---CKVILVM FNYFIMANFF DALLFSDDET TDCSTQP-SL M-------- --GCKAVMVL FNYFVMANEF DTLLFSGEQN AECSAQP-SL VRS------ --GCKVVLVF FNYFVMA-TF DDILEN--RT SQCSNQP-SL AG------- ---CKASLVF FQYFIMANFF DTLLFSEDEA TDCSTQP-SL V-------- --GCKATLVF FNYFVMANFF DDI LFN--KT LQCSNQS-SL LG------- ---CKASLVF FQYFIMANFF DDILYSSSNT AHCPEDQTSW VG------- ---CKAILVF FQYGVMANFY DDVLYSSSGT LHCPDQPSSW VG------ --CKLSLVF LQYCIMANFF $\star::: \quad{ }^{\star} .{ }^{\star} .{ }^{\star} \quad:::::^{\star} \quad::^{\star} \quad:$ $\left.\left.\overline{\cdots|\cdots|}_{260} \ldots|\ldots|\right|_{270} \ldots|\ldots|\right|_{280} \ldots|\cdots|_{290} \cdots|\ldots|$ WLLVEGLYLH TLLMVIFSEN RHFIIYLLIG WGFPTVEVTP WIVCRIYLED WLLVEGLYLH TLLMVIFSEN RHFIIYLLIG WGFPTVFMIP WIVCRIYLDD WLLVEGLYLH TLLLIIRNAS IRLPVYMLIG WGIPFVFMVA WVICRVNLED WLLVEGLYLH TLLVVIFSEN RHFIVYLFIG WGIPAVFVFV WVMMRIYLED WLLVEGLYLH TLLLVTYAY- THLAVYLTIG WGLPSVFLVV WVFCRIYLED WLLVEGLYLH TLLLIIRHSS IRLSVYMLIG WGIPFVFTLA WVICRINLED LAAVEGLYLT RYRESERSSS CRVA----- -GIPLVFMAA WIACRINLED WLLVEALYLH TLLVVIFSEN RHFVVYMFIG WGIPTVFVSA WVMTRIYLED WLLVEGLYLH TLLLVIHTYS TRLSIYMFIG WGIPFVFVVA WIISRVNLED WLLVEGLYLH TLLVVIFSEN RHFIIYMFIG WGIPTVFVSA WVITRIYLED WLLVEGLYLH ILLVLIFSPN RHFTVYLLIG WGIPTIFIIT WTVTRIILED WLLVEGLYLH TLLVAMLPPR RCFLAYLLIG WGLPTVCIGA WTAARLYLED ${ }_{\star \star *}^{* \star \star}$. ${ }^{*}:{ }^{*}:{ }^{\star}:{ }^{*}:{ }^{*}$

TMD5

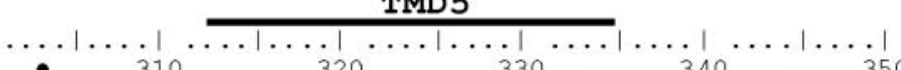

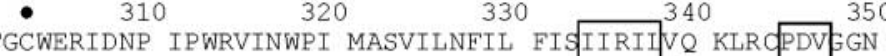
TGCWERNDAP IPWRVINWPI MASVILNFIL FISIIRIIVQ KLRQPDVGGN TRCWEMNENP VPNRLINWPI MASVIINFIF FISIIRIIVQ KLQ AADVFGN TGCWETNDNP IPNWVINGPI GFSIMVNFLL FVSIIRIIVQ KLRQPDVEGN TGCWERNDIP TPWRVINWPI MASVIINFVL FISITRIIVQ KLROSDVFGN TGCWEKHENP VLSRLIHWPI MASVIINFIL FISIIRIIVQ KLRQTDVEGN TQCWEMNENP IANRLMNWPI MASVMINFVL FVSIIRIIVQ KLROPDVEGN TGCWERNDNP IPNWVINGPI GFSIMVNFIL FISIIRIIVQ KLRQPDVEGN TSCWEINDNP VPDRLINWPI MASIIINFIL FISIIRVIVQ KLROSEVEGN TGCATTSD-- ----ICKSSI WFFIRVNFIL FISIIRIIVQ KLROPDVEGN TGCWDTNEHG GPWWVIRIPI LISIIVNFIL FISIIRIILQ KLRSPDVFGN TGCWDTNDHS VPWWVIRIPI LISIIVNEVL FISIIRIILQ KLTSEDVGGN

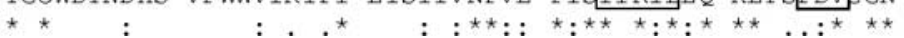




Zebrafish PHIR
Goldfish PHIR
Fugu VPAC2RA
Fugu VPAC2RB
Trout VPAC2R
Tetraodon VPAC2R
Stickleback VPAC2RA
Stickleback VPAC2RB
Medaka VPAC2RA
Medaka VPAC2RB
Chicken VPAC2R
Human VPAC2R
Clustal consensus

Zebrafish PHIR Goldfish PHIR Fugu VPAC2RA Fugu VPAC2RB Trout VPAC2R Tetraodon VPAC2R stickleback VPAC2RA stickleback VPAC2RB Medaka VPAC2RA Medaka VPAC2RB Chicken VPAC2R Human VPAC2R Clustal consensus

\begin{tabular}{|c|c|c|}
\hline & TMD 6 & \\
\hline & 370 & $\begin{array}{l}\cdots 1 \\
400\end{array}$ \\
\hline DQSQYRRLAK & STLLLIPLFG VHYIVFVYII & DGNGERE-NY KIFFDLGLG- \\
\hline DQSQYARLAK & STLLLIPLFG INYVVFVYII & EENEEMN-NY KIFFDLGLG- \\
\hline EQSQFARLAK & STLLLIPLFG INYVVFVYMM & EPSDTNLNYV KIFFELGLG- \\
\hline DQSQYARLAN & STLLMIPLFG IHYVIFVTLG & ESIAE---DY KIFFDLALG- \\
\hline DQSQYHRLAK & STLLLIPLFG VNYMVFVYLV & ETESDGMEEY KILFDLVLG- \\
\hline KKSQFARLAK & STLLLVALFG INYVVFVYTM & EPSDMNLRYA KIFFDVGLGS \\
\hline EQSQYARLAK & STLLLIPLFG INYVLFVYLM & EPADTNLRHV KIFFELALS- \\
\hline DQSQYARLAK & STLLLIPLFG IHYVVFVSLS & ESIAE---AY KIFFDLALG- \\
\hline DQSQYARLAK & STLLLIPLFG VHYVVFFYLM & EPDDQILKQI KIFFDLGLG- \\
\hline DQSQYHRLAK & STLLLIPLFG IHYVVFVSFS & ESITE---DY KIFFDLALG- \\
\hline DQSQYFRLAK & STLLLIPLFG VHYTVFALFP & DRSSN---NY KII FELCLG- \\
\hline DQSQYRRLAK & STLLLIPLFG VHYMVFAVFP & ISISS---KY QILFELCLG- \\
\hline $0+t+t+t$ & $\star \star \star \star ⿱ 亠 乂: .^{\star \star \star}::^{\star} \quad:{ }^{\star}$ & $\quad{ }^{\star}:{ }^{\star}::{ }^{\star}$ \\
\hline
\end{tabular}

TMD7

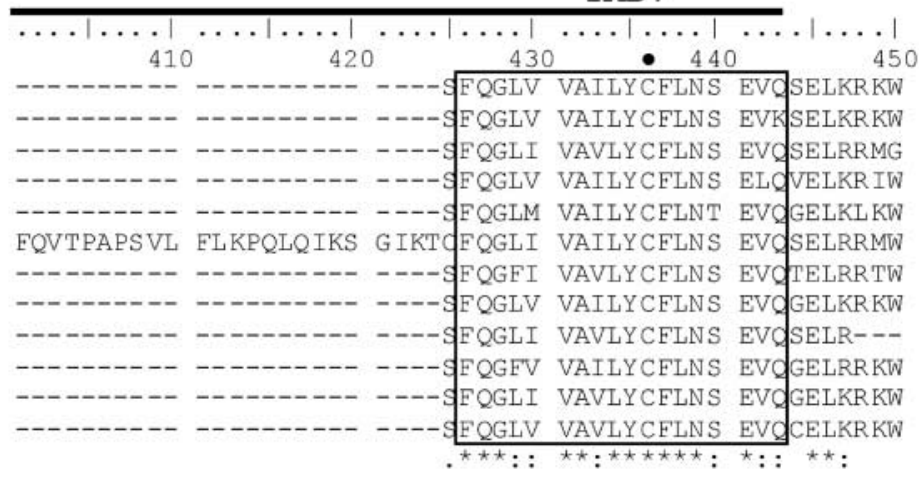

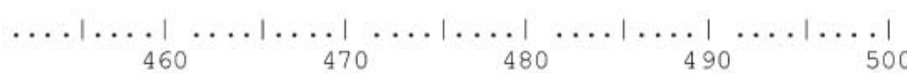
RSLRLKRYIG RDYRLHSSSI SRSGTENMAQ EQRNTRAQSE LQTETTVV-RSLRLKRYIG RDYRLHSSST SRNGTENMGR FHRNSK--- - - RSLSLKRYVG RDYKLHAASA SPNGGESSAR LRELIEVVCT ENRPDSSLKR RIMCLNAHLS G-HHSNSTFR S--GSELMAQ SHRNSRAQS I MOSETTVL-RSVSLKRYMW RDYRLHRGSI SHNGTDNSTQ EQRNSRAQSI LQTETTSL-RSLSLKRYMG QDDKMLAVSA GRN-TETSAQ FPRSFRAQS I LQTETSML--

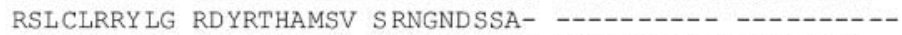
RSVCLNCRLS RDQHLHGTFA SRNGSEHAAQ FHRNSRAPS I LQSETTVL-RSLSLKRCVG REEKLQAVTL NRNGSQRS-- - RSMCLNCRLS RT PHFSSTET SRNGSEHMMQ EHRNSRAQS I LQSETTML-RSLCWKQTAG RDYRLH-SSI SRNGSESVSQ LHRNSRAQSE MQTETTMI-RSRCPTPSAS RDYRVCGSSF SRNGSEGALQ EHRGSRAQSF LQTETSVI --

Figure 1 (A) Predicted structure of zebrafish PHIR gene from zebrafish genome database in Vega. The gene size ( $>79 \mathrm{~kb})$ is indicated on the right of the gene. Exons are numbered and are shown as closed boxes; a solid horizontal black line indicates introns. The distance between the first five exons is indicated by a number above the intron line. (B) ClustalW alignment of amino acids of zebrafish PHIR with other known PHIR and VPAC2Rs. The transmembrane domains are annotated above the sequence. Thirteen conserved cysteines are marked with a black dot, although fugu VPAC2RB, stickleback VPAC2RA, and medaka VPAC2RA each lack one cysteine. Two potential conserved $N$-glycosylation sites (Asn-X-Thr/Ser) in the N-terminal extracellular domain for zebrafish are labeled with a black triangle. Opened circles or ovals show where Asn is not conserved. All signature motifs for both PHIRs and VPAC2Rs are in open boxes.

highest match is with goldfish PHIR. Also, the PHIR shares high sequence identity with trout VPAC2R $(65 \%)$, other teleost VPAC2Rs (47-61\%), chicken VPAC2R (59\%), and human VPAC2R (56\%; Table 4). For other members of the secretin receptor family, zebrafish PHIR shares sequence identity with zebrafish PAC1R-short $(42 \%)$, VPAC1R (42\%), GHRH-LPR (37\%), and GHRHR (35\%). The percent identity plus the functional study suggests that the present cDNA encodes VPAC2R a PHIR, not a VPAC2R. Hereafter, we refer to the receptor as PHIR. 
The full-length cDNA of the PHIR blasted against the zebrafish genome database (Vega Multi Blast View) shows that the zebrafish PHIR gene spans more than $79 \mathrm{~kb}$ on chromosome 24 and consists of 14 exons (Fig. 1A). Sequence alignment of PHIRs and VPAC2Rs from various species (Fig. 1B) indicates that zebrafish PHIR has amino acid signature motifs such as PDV and IIRIL characteristic of VIP binding receptors, and has RLAK between TMD5 and TMD6 for coupling to $G_{s}$
(Chow et al. 1997, Cardoso et al. 2004). Another amino acid motif, the highly conserved region for the mammalian secretin B family members at TMD7, FQGBBVXXBYCFXNXEXQ (Lok et al. 1994), was identified where $X$ represents any amino acid residue and $\mathrm{B}$ represents any hydrophobic amino acid residue (Fig. 1B). In addition to these motifs, two conserved residues, $\mathrm{R}$ and $\mathrm{K}$, localized within TMD2 and associated with VPAC1R activation (Solano et al. 2001)
ZFGHRH-LP R z FGKRH-LPR * GFGHRH-LPR ҒUGKRH-LP R ChGHRH-IP R clustal cons

Z FGHRH-LP R zfGKRH-LPR * gfGKRH-LPR fUGHRH-LPR ChGHRH-LPR Clustal cons

z FGHRH-LPR z fGHRH-LPR * g fGHRH-LPR FUGHRH-LPR ChGHRH-LPR clustal cons

z FGHRH-LPR Z FGHRH-LPR * gfGHRH-LPR fUGKRH-LPR ChGHRH-LPR Clustal cons

Z FGHRH-LPR z FGHRH-IPR * gfGHRH-LPR ҒUGHRH-LPR ChGHRH-LPR clustal cons

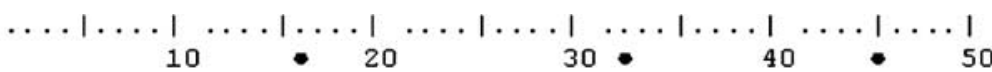
--.--M TUVHLCLFAG FWRAMHAIHP DCAIISQLIQ DQETCIOTRK 作

MFCVLGFN-M TVLHLCLFAT VWRAVQAIHP ECDIISQFIQ DOEICIQTRE MCCLERQEKF TVLIFCLHLW HTPTAQAIHP DCAIISAHQR AQEMCKQTRR ---MENAE GMPHCRPFQN PLPAARDKEQ GLNAPSLVAQ GASASKPTRQ

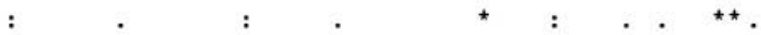

$\ldots|\ldots| \ldots|\ldots| \ldots|\ldots| \ldots|\ldots| \ldots|\ldots| \ldots|\ldots| \ldots \mid$

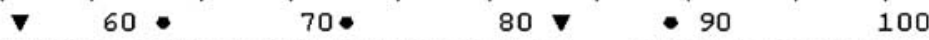
REIKNHSQQT GCGTDWDGIR CWHTANTGQL IN---VSCSD VFQHIS-NTQ - - - - - - - - - - - - - - - - REIKNQSQQT GCWTDWDGIR CWQTAKTGQL IN---VSCSD VFQHIS-NTQ SEAQNQTNQS -CTTLWDNIR CWPWAEVGQV VN---VSCAE VLQDF S-SNQ -EQPVLOLRT FLQKFKEVVG IHPECKIFQQ VVKEEALCLE RNESASPDLK

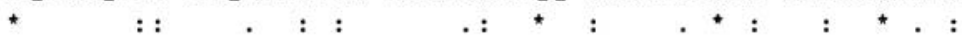
$\ldots|\ldots| \ldots|\ldots| \ldots|\ldots| \ldots|\ldots| \ldots|\ldots| \ldots|\ldots|$ T. $110 \quad 120 \quad * \quad 130 \quad 140 \quad 150$ GFIYRNCVSN G-WTDPYPPY EEACAFEDDS E--SGTVTTY LSTLKQLYTA GFIFRNCTKD G-WTEVYPSY ENACEIVEDV E--SETETTY YATFRQVYTA GFIYRNCSSN G-WSDPYPSY EEACTFEDDS E--SGTVSTY LSTLKQLYTA GFUYRNCTAD G-WSELYPPY ORACAVRNDS E--PESETSY LATFRQIYTV GF LQRNCTQE AYWSEPFPSY AVACGFDEGS SKGPEDOKSY YSAFWRVYTA

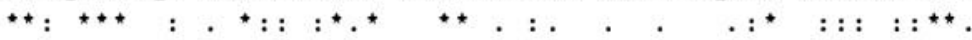

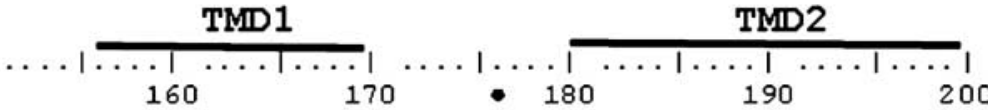

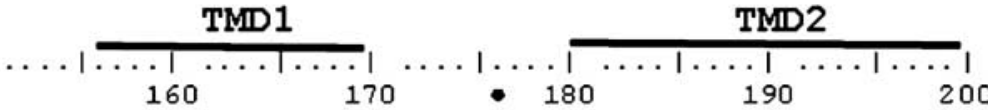

GYATSLISLI TAVIIFTCFR --KFHCTRNYIH INLFUSFILR ASAVFIKG GYATSISLI TAIFVFTVFR NRKFHCTRNYIH INLFASFILR ASAIFIKD GYATSLISLI TAVIIFTCFR --KFHCTRNYIH INLFVSFILR ATAVFIKD GYATSLITLI TAIVUFTAFR --KFRCTRNYIH VNLFSSFILR ASAVFIKD GYAASUTSLI TALIVFAAFR --KFHCTRNYIH MHLFVSFILR AIAVFTKD

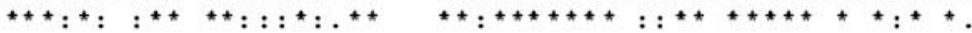

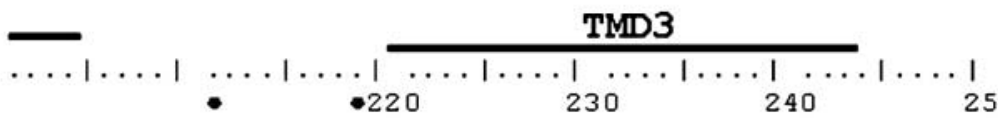

TULFSDETQDHC LMSTULCKSA VTFFOFCILT NYFWLLVEGL YLQTLLALT AVLFADENLDHC FMSTTSCKAA VAFFOFSILA NYFWLLVEGM YLQTLLALT AVLFSDETQNHC LMSTVACKTA UTFFOFCILT NYFWLLVEGL YLQTILTLT TVLFADESLDHC SMSTTACKSA VAFFOFSILA NYFWLLVEGM YLQTLLALT AVLFADETMDHC LMSTVACKAA VAFFOF SILA NFFWLLIEGI YLQTLLLLT

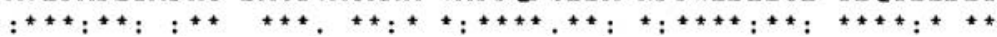

Figure 2 (continued) 


\section{z FGHRH-LPR z FGHRH-LP R* gfGHRH-LPR fUGHRH-LPR ChGHRH-LP R Clust al cons}

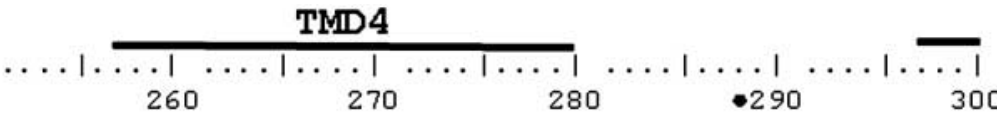

FVSQRKYFWWY ILIGWGVRSV VLVUWULTRQ FYDNRGCWDD TDNTNIWWI FV SQKKYFWWY ILIGWGLPTL ILTIWULARN FFDNNGCWDD TDVAYFUTI FVSQRKYFWWY ILIGWGVPSV VLIVWULTRQ FYDNRGCWDD TDNMNIUWI FVSQRKYFWWY ILIGWGLPSA VLVLWULTRF IYDNRSCWDD TDNVAIUTI FVSDKQYVWWF IFAGWGAPTA VML TWVLTRL HQQNTGCWDD DENGVVLWI

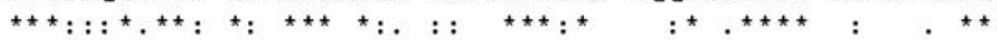

\section{TMD5}

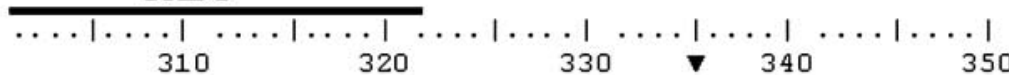

zfGHRH-LPR zfGHRH-LPR * GFGHRH-LPR fUGHRH-LPR ChGHRH-LP R clust al cons

\section{Z FGHRH-LP R z fGHRH-LPR * gFGHRH-LPR fUGHRH-LPR ChGHRH-LP R Clust al cons}

IKGPITASLFT NIIIFLNVIR ILVQKLKSPG VGGN-DTGHF M----IKGPITVSLLI NFLIFINVIR ILVQKLKSPG MGGG-DTNHF ILLSFTVLA IKGPITVSLIA NIIIFLNVIR ILVQRLKSPG VGGN-DTGHF M-----IKGPITVSLLV NILIFINVIR ILVQRLKSSA MAGNHDTGHY M-----IKGPILLTVLI NFIIFINVIR ILVHKLKSQE GGGS-NSSHF V------

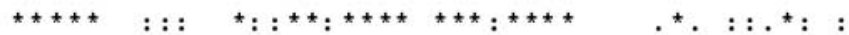

\section{TMD6}

$$
\begin{aligned}
& \text { TMD7 }
\end{aligned}
$$

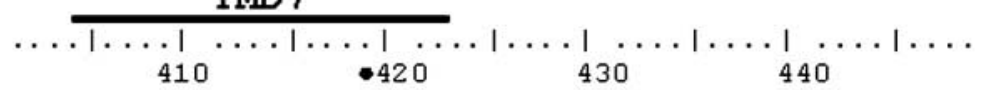

YIELGLGSFQ GFVVALLYCF LNGEVQAELK RRLWTWLTQT HLSPCKKRR FLELGLGPFQ GFVVALLYCF LNGEVQAELK KRLWKWQTQS YLRYSKRRR YIELGLGSFQ GFVVALLYCF LNGDVQAELK RRLWTWQTQT HLSPSKKRQ YIELGLGSFQ GFVVALLYCF MNGEVQTELR RWLRKCCNQN HPTQAKR-YIELGLGSFQ VSRTKSL--- - - $:: * * * * * * \quad$. *

\section{z FGHRH-LPR \\ ZFGHRH-LPR* gfGHRH-LPR \\ fUGHRH-LPR ChGHRH-LPR clust al cons}

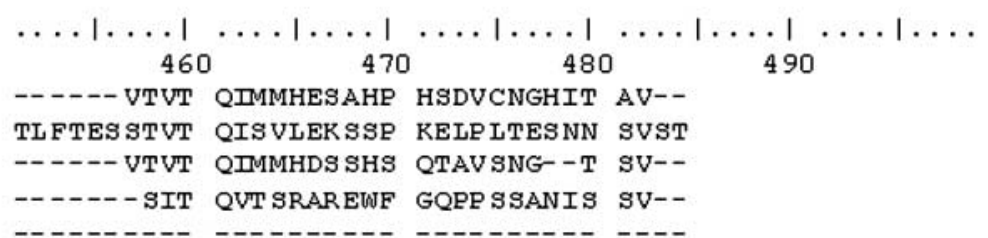

Figure 2 ClustalW alignment of amino acids for two zebrafish (zf) GHRH-LPRs (*the truncated form) with goldfish (gf), fugu (fu), and chicken (ch) GHRH-LPRs (a.k.a PACAP-related peptide receptor or PRPR). The transmembrane domains are annotated above the sequence. Thirteen conserved cysteines in the fish receptors are marked with black dots and three potential conserved $N$-glycosylation sites in the $\mathrm{N}$ terminal extracellular domain in fish are labeled with a black triangle. Signature motif 'RLAK' is shown by an open box. As noted, chicken $\mathrm{GHRH}-\mathrm{LPR}$ has only three conserved cysteines and one glycosylation site in the N-terminus compared with the GHRH-LPR of fish. 
were identified. Thirteen conserved cysteines, which are involved in tertiary structure and ligand binding, were also found in the zebrafish PHIR. There are only two conserved putative glycosylation sites in PHIRs in zebrafish at N-terminal extracellular sites in contrast to three conserved sites in those of chicken and mammals. However, goldfish PHI receptor along with the zebrafish PHIR and fugu putative VPAC2RA and VPAC2RB have only two glycosylation sites (Asn-X-Ser/Thr, where $\mathrm{X}$ is any amino acid except proline) in the $\mathrm{N}$-terminal extracellular domain.

The zebrafish GHRH-LPR cDNA is 1747 bp in length (GenBank, accession No. EU150382) with an open reading frame of $1296 \mathrm{bp}$ (from 181 to 1476 ) encoding a 432 amino acid protein (Fig. 2). A Kyte-Doolittle hydrophobicity plot (data not shown) of the deduced protein was used to identify seven putative transmembrane domains. The amino acid sequence of the zebrafish GHRH-LPR shares the highest level of identity with goldfish GHRH-LPR $(88 \%)$, followed by fugu $(64 \%)$, and chicken $(43 \%)$ as seen in Table 5 . On the other hand, zebrafish GHRH-LPR only shares 39-40\% sequence identity with GHRHR of zebrafish, goldfish, chicken, and human. The intron and exon organization was not revealed by a BLAST search of the fulllength cDNA of GHRH-LPR in the zebrafish genome database (Vega Multi Blast View) as the genome data are not complete. Sequence alignment of GHRH-LPRs from various species (Fig. 2) revealed that zebrafish GHRH-LPR is very similar and structurally related to receptors of other fish. Thirteen conserved cysteines and three putative glycosylation sites, and signature motifs like RLAK, FQGBBVXXBYCFXNXEXQ and SQR were observed in zebrafish, fugu, and goldfish receptors.

The zebrafish PHI/VIP cDNA is $1092 \mathrm{bp}$ in length (GenBank, accession no. EU150383) with an open reading frame of $459 \mathrm{bp}$ (from 117 to 575) encoding a 153 amino acid precursor. The genomic database in Vega revealed that the PHI/VIP gene spans $\sim 3584 \mathrm{bp}$ on chromosome 13 and contains six exons (Fig. 3A and B). The zebrafish mature PHI peptide shares high sequence identity with goldfish PHI A (85\%) and B (89\%), chicken PHI $(67 \%)$, and human PHM $(78 \%)$ peptides (Tse et al. 2002; Fig. 3C). The zebrafish VIP is identical with the mature VIP peptide of trout. Both PHI and VIP in the zebrafish precursor cDNA are flanked at the C-terminus by a GKR, indicating that the peptides are amidated and have potential proteolytic cleavage sites (Fig. 3B).

\section{Phylogenetic analysis and synteny comparisons}

Phylogenetic analysis involved zebrafish PHIR and GHRH-LPR with other members of the secretin receptor superfamily. Analysis of all ClustalW multiple aligned receptors (TMD 1-7) was conducted by the ML method and the NJ method with the GLUCR as the outgroup. The ML tree (Fig. 4) showed a similar result as the NJ tree (Supplementary Figure 1, see Supplementary data in the online of version of the Journal of Endocrinology at http://jme.endocrinology-journals. org/content/vol41/issue/5). The analysis indicates that the five major groups of secretin superfamily receptors included the GHRHR, GHRH-LPR, PAC1R, VPAC1R, and the PHIR/VPAC2R; each group had a bootstrap value of 99-100. The identified zebrafish GHRH-LPR was in a subgroup with like receptors and the potential for grouping with GHRHRs (bootstrap value of 66 in NJ tree but only 46 in ML tree). The PHIR/VPAC2R group had three subgroups: 1) zebrafish and goldfish PHIRs were in a subgroup with five other teleost VPAC2RAs (bootstrap $=100), 2$ ) three teleost duplicate VPAC2Rs (receptor B, bootstrap $=75$ ) for fugu, medaka, and stickleback were together, and 3) chicken and human VPAC2Rs formed the third subgroup (bootstrap $=96$ ).

The chromosomal location of the PHIR gene or VPAC2R in zebrafish, medaka, chicken, and human are shown in Fig. 5. Two linked genes in the same chromosomal locations are also shown. In zebrafish, the PHIR gene is found on chromosome 24 along with two linked genes (zmynd11 and xr_029619.1 with the latter being similar to human DIP2C). The same arrangement is present for medaka on chromosome 17 and for chicken on chromosome 2. However, in human the VPAC2R gene on chromosome 7 has been separated from the linked genes on chromosome 10 .

\section{Functional analysis of PHIR compared with related zebrafish and human receptors}

To evaluate whether the identified PHIR and PHI peptide are able to transduce a physiological signal, COS7L cells were separately transfected to express zebrafish PHIR, VPAC2R, PAC1R-short, and human VPAC2R. The ability of zebrafish peptides to activate adenylyl cyclase and phospholipase $\mathrm{C}$ pathways was assessed by the cAMP and $\left[{ }^{3} \mathrm{H}\right]$ IP assay (Fig. 6). In COS7L cells expressing the PAC1 receptor, three peptides but not $\mathrm{PHI}$ were able to activate the cAMP and IP accumulation in a dose-dependent manner with different half-maximal response $\left(\mathrm{EC}_{50}\right)$ values (Fig. 6A and B). There was no significant difference $(P<0 \cdot 05)$ in $\mathrm{EC}_{50}$ values of PAC1R stimulation by the two different PACAP peptides in either the cAMP or IP pathway. But the $\mathrm{EC}_{50}$ values of the PAC1R response to VIP were significantly higher $(P<0 \cdot 05)$ than those of the PACAP peptides in either the cAMP or IP pathway. Hence, zebrafish PACAP was more potent than zebrafish VIP on the zebrafish PAC1R.

In cells expressing the VPAC1R, the same three peptides (PACAP1, PACAP2, VIP) were able to activate the cAMP pathway but PHI was not active in a 
Table 5 Percentage of amino acid identity of aligned zebrafish growth hormone-releasing hormone-like peptide receptor (GHRH-LPR) with others in the same gene family

\begin{tabular}{|c|c|c|c|c|c|c|c|c|}
\hline & $\begin{array}{l}\text { Zebrafish } \\
\text { GHRH- } \\
\text { LPR }\end{array}$ & $\begin{array}{l}\text { Goldfish } \\
\text { GHRH- } \\
\text { LPR }\end{array}$ & $\begin{array}{l}\text { Fugu } \\
\text { GHRH- } \\
\text { LPR }\end{array}$ & $\begin{array}{l}\text { Chicken } \\
\text { GHRH- } \\
\text { LPR }\end{array}$ & $\begin{array}{l}\text { Zebrafish } \\
\text { GHRHR }\end{array}$ & $\begin{array}{l}\text { Goldfish } \\
\text { GHRHR }\end{array}$ & $\begin{array}{l}\text { Chicken } \\
\text { GHRHR }\end{array}$ & Human GHRHR \\
\hline
\end{tabular}

Amino acid identity was determined by using the ClustalW alignment program with BLOSUM62. The second form of zebrafish GHRH-LPR is not included, as it lacks 100 amino acids in the $\mathrm{N}$-terminal domain compared with the receptors listed above.

physiological range. None of the peptides tested were able to stimulate VPAC1R in the IP pathway in the physiological range $\left(<10^{-6} \mathrm{M}\right.$; Fig. $6 \mathrm{C}$ and D). PACAP and VIP are nearly equal in potency for the stimulation of the zebrafish VPAC1R $\left(\mathrm{EC}_{50}=1 \cdot 5-3 \times 10^{-7} \mathrm{M}\right.$; Table 6) but the potency of the three peptides is greater for the PAC1R than for the VPAC1R.

PHIR had a different response pattern to PACAP and VIP compared with zebrafish VPAC1R or VPAC2R from other species. The PHIR had no significant response $(P>0.05)$ to VIP, PACAP1, or PACAP2 in either the cAMP or IP pathways (Fig. 6E and F). In contrast to VPAC1R or VPAC2Rs, VIP does not stimulate the zebrafish PHIR, although the latter has high identity to VPAC2Rs of other species. However, the PHIR had a high potency in response to $\mathrm{PHI}$ in the cAMP assay with an $\mathrm{EC}_{50}$ value of $1.77 \times 10^{-9} \mathrm{M}$ (Fig. 6E). Hence, the PHIR is coupled to the $G_{s}$ protein that activates the adenyl cyclase pathway but is not coupled to $G_{q / 11}$ protein that activates the IP pathway. Also, PHIR is distinct from the VPAC1R and VPAC2R in two ways: it is activated by PHI and is not activated by PACAP or VIP.

The physiological characteristics of the zebrafish receptors were compared with human VPAC2R using our zebrafish VIP and human VIP, two peptides with $82 \%$ identical amino acids. Zebrafish VIP was able to activate the human VPAC2R in both the cAMP (Fig. 6G) and IP (Fig. 6H) signaling pathways in a dosedependent manner. It was striking in the cAMP pathway that human VIP and zebrafish VIP produced similar $\mathrm{EC}_{50}$ values with the human VPAC2R $\left(7 \cdot 6 \times 10^{-9} \mathrm{M}\right.$ in response to human VIP and $5 \cdot 4 \times 10^{-9} \mathrm{M}$ in response to zebrafish VIP; Table 6). Unexpectedly, zebrafish VIP also stimulated the human VPAC2R in the IP signaling pathway (Fig. $6 \mathrm{H}$ ), as this VPAC2R primarily activates the cAMP path. VIP did not stimulate the zebrafish VPAC1R in the IP path as expected. Hence, zebrafish VIP is a potent stimulus for the human VPAC2R.

Also, the human VPAC2R was stimulated by zebrafish PHI. The $\mathrm{EC}_{50}$ was $7 \cdot 4 \times 10^{-9} \mathrm{M}$, which was not significantly different from the $\mathrm{EC}_{50}$ for VIP suggesting a potent response (Fig. 6G). Part of the effect may be explained by the high sequence identity $(78 \%)$ between zebrafish PHI and human PHM peptides, although zebrafish PHI was even more potent than human PHM $\left(\mathrm{EC}_{50}=4.7 \times 10^{-8}\right)$ with the human receptor. In short, zebrafish PHI and VIP are potent stimulators of the human VPAC2R.

\section{Functional analysis of zebrafish GHRH-LPR and human GHRHR}

To confirm the functional identity of zebrafish GHRHLPR, we used three peptides: zebrafish GHRH-LP1, GHRH-LP2, and true GHRH. Each peptide was tested on the zebrafish GHRH-LPR expressed in COS7L cells. For comparison of the physiological profiles, human GHRHR was also expressed in COS7L cells. Both cAMP and IP production were measured after stimulation with different doses of peptides. Zebrafish GHRH-LPR responded to zebrafish GHRH-LP1 $\quad\left(\mathrm{EC}_{50}=3 \cdot 3 \times\right.$ $\left.10^{-8} \mathrm{M}\right)$ and to zebrafish GHRH $\left(1.2 \times 10^{-7} \mathrm{M}\right)$ with no significant difference $(P>0 \cdot 05)$; the responses in the cAMP signaling pathway were dose dependent (Fig. 7) and similar to those in the chicken or goldfish system (Lee et al. 2007, Wang et al. 2007). However, there was no response of zebrafish GHRH-LP receptor to zebrafish GHRH-LP2 stimulation; likewise the goldfish receptor only responded to one of its two GHRHlike peptides (Lee et al. 2007). We found that human GHRHR had no response to either zebrafish GHRHLP1 or zebrafish GHRH-LP2 but did respond to human GHRH as a positive control.

\section{Tissue expression of zebrafish PAC1R, VPAC1R, PHIR, GHRH-LPR, GHRHR, and PHI/VIP mRNAs in adults}

To investigate the expressions of zebrafish PHIR, GHRH-LPR, and PHI/VIP mRNAs in comparison with 
zebrafish PAC1R, VPAC1R, and GHRHR mRNAs, RT-PCR was used for 11 tissues (Fig. 8). Zebrafish $\beta$-actin cDNA (1014 bp) was used as a control to verify the quality of the first-strand cDNA synthesized. The most widely distributed receptor is PHIR mRNA, which is expressed strongly in all tissues. The VPAC1R-short mRNA (arrow in Fig. 8) was also widely expressed in adult zebrafish with stronger expression in the eye, brain, testis, gill, skin, and ovary; lower levels were detected in the heart, kidney, swimbladder, skin, and

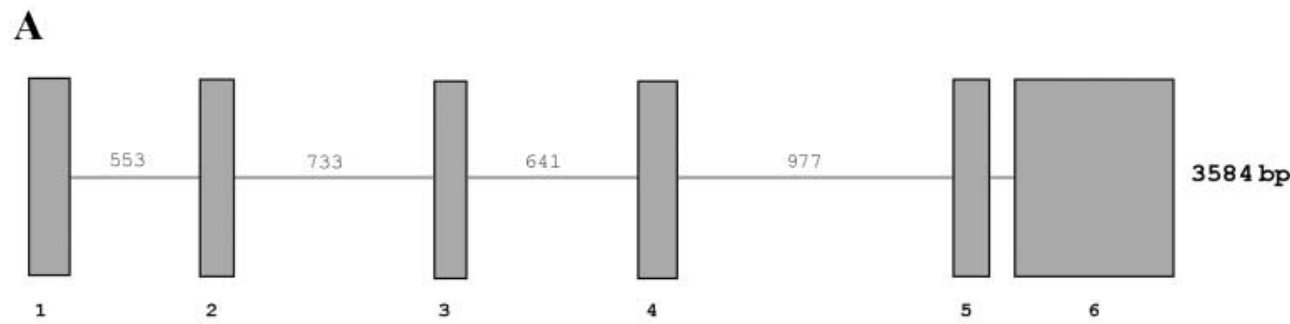

B

1 AAAGAGACACAAGAGCGCCA.AAGGAACT TAAAAAGTCCACCTGGAGACTTTGGGAGAT TTGACTGGAGG 70

71 TATTTCAGCACCCGATCAGAGCGCGGATCTCACATCTCCA TTGCACATGTGT A.AGCA. TGCT TGTGCGG 140 $\begin{array}{lllllllllll} & \mathrm{C} & \mathrm{C} & \mathrm{K} & \mathrm{A} & \mathrm{M} & \mathrm{L} & \mathrm{V} & \mathrm{R}\end{array}$

141 AACGCC TCTCAGCTCTTGCTCTTCA TAACTCTT TCCAGTGTTTT ATATGCCCGGACCTT AAGT TTACCCT 210

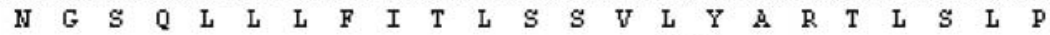
PHI

211 TCGCCT CCAT GAGAGAAACAAGACACGCAGACGGCCTCTTCACAAGCGGATACAGTAAACTTCTAGGACA 280 \begin{tabular}{llllllllllllllllllllllllll} 
& $\mathrm{A}$ & $\mathrm{A}$ & $\mathrm{S}$ & $\mathrm{M}$ & $\mathrm{R}$ & $\mathrm{B}$ & $\mathrm{T}$ & $\mathrm{R}$ & $\mathrm{H}$ & $\mathrm{A}$ & $\mathrm{D}$ & $\mathrm{G}$ & $\mathrm{L}$ & $\mathrm{F}$ & $\mathrm{T}$ & $\mathrm{S}$ & $\mathrm{G}$ & $\mathrm{Y}$ & $\mathrm{S}$ & $\mathrm{K}$ & $\mathrm{L}$ & $\mathrm{L}$ & $\mathrm{G}$ & $\mathrm{Q}$ \\
\hline
\end{tabular}

281 AT TATCTCCC AGACGGT ACCT GCAGTCAT TGATCGGAAAGCGCGTCAGTCATGATT TGA TGGA.AGACCAG 350

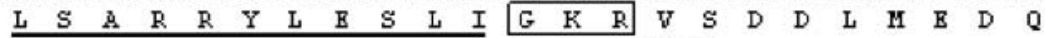
VIP

351 GCGCCGATGAACCGTCA TTCAGATGCA.ATATCACAGACAACTACAGTCGCT TTCGCAAGCAGATGGCGG 420 $\begin{array}{llllllllllllllllllllllll}A & \mathrm{P} & \mathrm{H} & * & \mathrm{~K} & \mathrm{R} & \mathrm{H} & \mathrm{S} & \mathrm{D} & \mathrm{A} & \mathrm{I} & \mathrm{F} & \mathrm{T} & \mathrm{D} & \mathrm{N} & \mathrm{Y} & \mathrm{S} & \mathrm{R} & \mathrm{F} & \mathrm{R} & \mathrm{K} & \mathrm{Q} & \mathrm{M} & \mathrm{A}\end{array}$

421 TGAAGAAATA TCTGAAC TCTGTTCTCACAGGAAACCGAAGTCAAGAAGACCCACCCAGT ATGCAGGAGGA 490 $\begin{array}{lllllllllllllllllllllllll}\mathrm{V} & \mathrm{K} & \mathrm{K} & \mathrm{Y} & \mathrm{L} & \mathrm{N} & \mathrm{S} & \mathrm{V} & \mathrm{L} & \mathrm{T} & \mathrm{G} & \mathrm{K} & \mathrm{R} & \mathrm{S} & \mathrm{Q} & \mathrm{E} & \mathrm{D} & \mathrm{P} & \mathrm{P} & \mathrm{S} & \mathbf{M} & \mathrm{Q} & \mathrm{E} & \mathrm{E}\end{array}$

491 ATCACCCGGCGGAGAAACCACATATCGGGAGAGCTACGATGACGTGACTGTAGACCGACTACTCAACCAT 560 $\begin{array}{llllllllllllllllllllllll}S & A & G & G & B & T & T & Y & R & B & S & Y & D & D & V & T & V & D & R & L & L & N & H\end{array}$

561 ATACCATTGCCGCTCTGAGGT CAGTCCGAACGCAAGAAAT CAAGACCATCCT TGGGTTTCCAT TTTACAC 630 I p L P L *

631 AGTCTAGCAT AGGACATCACCATACTACT ABAACAACGTGTTGAGGAABAAATAATATAATGTGGT TAC 700

701 CTATGACGTAAATGTTT GCAC TGTACATAGAAAMTAAACTATAATGAGAGATGTTATAGCTTGAAGATGT 770

771 ACATAGTGTGCTTT GGCCTTGGATCCGACACGT TTGCTTTGACATGTT TCCTGTATATTGTAGATCAATA 840

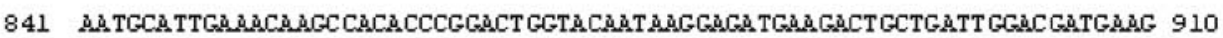

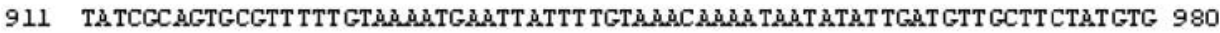

981 AACTCT AGAGGGACCAT TTGGGTTT TGGGTGGGTCGGGCA TTGGAAATCTTTATTT TTGCCAT TCAAAGA 1050

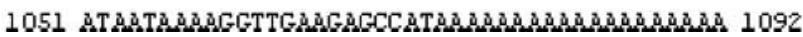

Figure 3 (continued) 


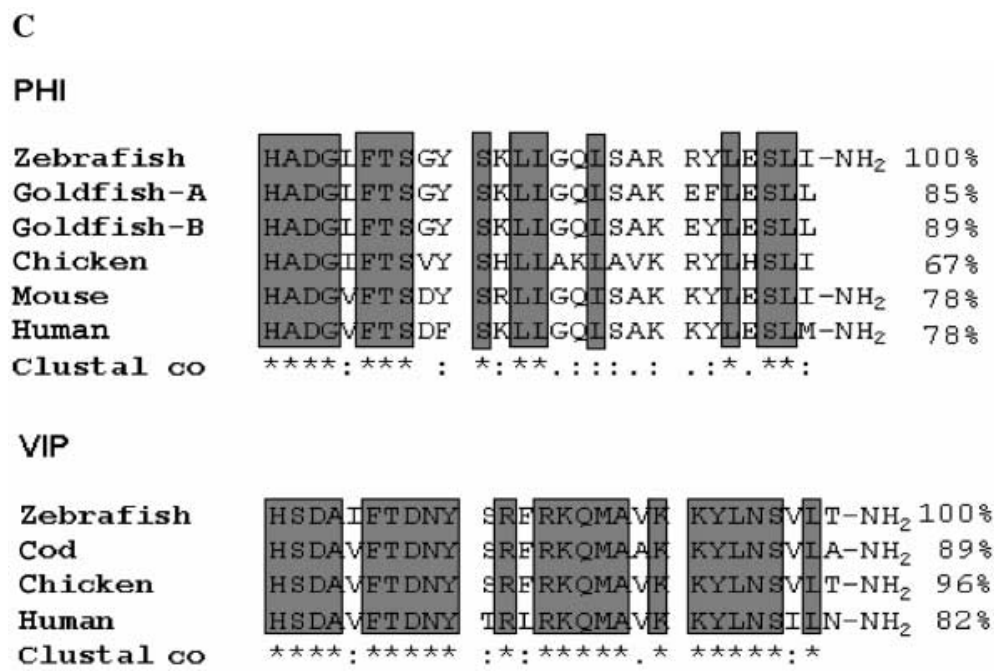

Figure 3 (A) Predicted structure of zebrafish PHI/VIP gene from the zebrafish genome data base in Vega blasted with our cDNA sequence. The gene size (3584 bp) is indicated on the right of the gene. Exons are numbered and are presented by closed boxes and a solid horizontal gray line indicates introns. The distance between the first five exons is indicated by numbers above the intron line. (B) cDNA and predicted amino acid sequences of zebrafish vip (PHI/VIP). The numbers on the right and left are the positions of the nucleotide sequences. The putative sequence of mature PHI and VIP are underlined and the putative PHM (C-terminal extension of PHI to the last residue (Met) before 'KR') is labeled with a black dot. The potential polyadenylation signals (AATAAA) are double underlined. (C) Amino acid sequence alignment of mature $\mathrm{PHI}$ and VIP peptides by ClustalW in vertebrates. Shaded areas represent identical amino acid residues. The sequences of goldfish A and B, chicken, mouse, and human are from Tse et al. (2002).

gut. The longer bands (arrow head in Fig. 8) are from PAC1R-hop mRNA, which has a hop cassette inserted in intracellular loop 3. PAC1R-short mRNA is more widely distributed than other forms of PAC1R mRNA in zebrafish (Fradinger et al. 2005). Also, PAC1R-short is the predominant isoform in goldfish (Kwok et al. 2006). VPAC1R mRNA was expressed in all 11 tissues with a lower level of expression in the gill, kidney, skin, and ovary. PHI/VIP mRNA was more strongly expressed in the eye, heart, brain, testis, gill, gut, and skin than in the swimbladder or ovary. GHRH-LPR and GHRHR mRNAs are distributed to only a few tissues. GHRH-LPR mRNA was only strongly expressed in eye, brain, testis, and kidney with very weak expression in swimbladder and heart. The GHRHR mRNA is mainly distributed in the brain, testis, and gut with very faint bands in the eye, kidney, and swimbladder. $\beta$-actin mRNA was expressed in a relatively similar manner in the 11 tissues.

\section{Discussion}

\section{Proof that a novel zebrafish receptor is PHIR and not VPAC2R}

Initially, we thought our newly isolated receptor of 2088 bp was a VPAC2R because it encoded a small section that was identical to a published fragment of VPAC2R (274 bp; Wang et al. 2003). Also, alignment of our deduced amino acid sequence with human and chicken VPAC2R showed a sequence identity of 56 and $59 \%$ respectively. Signature motifs characteristic of human and chicken VPAC2R were present in the zebrafish sequence: Thirteen conserved cysteines including seven in the $\mathrm{N}$-terminal extracellular domain, PDV, and RLAK binding motifs and other highly conserved amino acid residues for VIP binding (Fig. 1). Our phylogenetic analysis and chromosome linkage study also grouped this sequence with other VPAC2Rs.

In contrast to the mammalian VPAC2Rs, our receptor was not significantly stimulated by VIP even with a $10^{-5} \mathrm{M}$ concentration analyzed in both cAMP and IP signaling pathways. Also, in the N-terminal extracellular domain that is fundamental for VIP binding (Laburthe et al. 2003), the zebrafish sequence is missing a potential glycosylation site, which is expressed in the human, chicken, and frog VPAC2R (Hoo et al. 2001). $\mathrm{N}$-glycosylation sites are critical for ligand binding and correct delivery to plasma membrane of the human VPAC1R (Couvineau et al. 1995, 1996). However, the missing glycosylation site is not strong evidence for establishing the identity of our receptor, as the human VPAC1R functions correctly as long as two of the glycosylation sites are intact (Couvineau et al. 1996).

Finally, we discovered that our receptor has $80 \%$ amino acid sequence identity with goldfish PHIR; the goldfish sequence had been published (Tse et al. 2002) but not entered into the NCBI database. The goldfish 


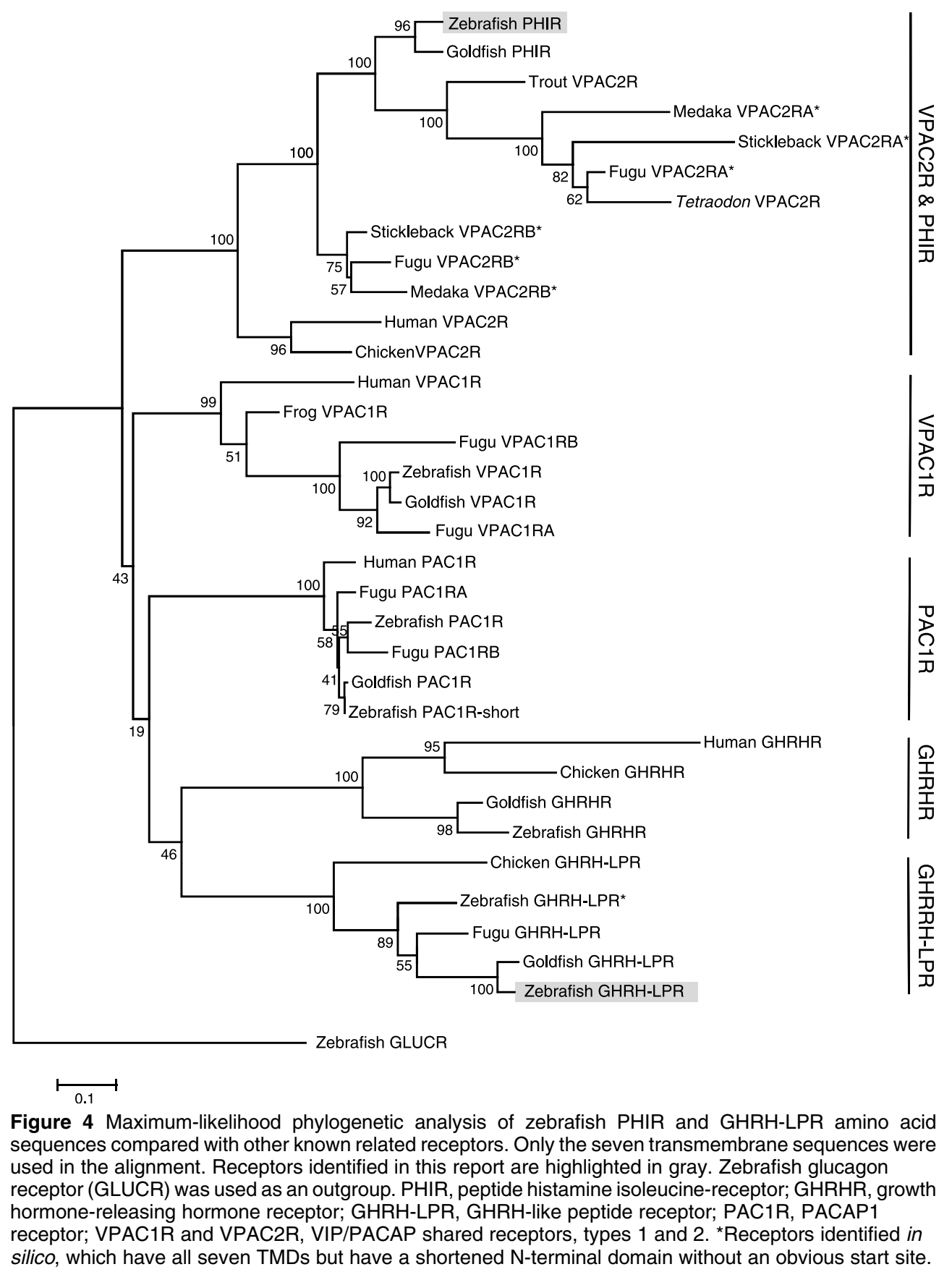

PHIR is the only one characterized in vertebrates so far as we know. The sequence identity of our receptor with goldfish PHI receptor is higher than that for all reported VPAC2Rs (up to 65\% in trout). Phylogenetic analysis also grouped the two fish PHIRs together. Moreover, in the N-terminal glycosylation site, goldfish has isoleucine in the same position as zebrafish (Fig. 1B; position 107, open circle) instead of asparagine needed for glycosylation. Based on functional studies (specific response to PHI and no response to VIP or PACAP), sequence identity, and phylogenetic analysis, we conclude that this receptor is zebrafish PHIR. Our strongest evidence comes from functional analysis with PHI in which the receptor was activated in a range expected for the natural ligand $\left(\mathrm{EC}_{50}=1.77 \times\right.$ $\left.10^{-9} \mathrm{M}\right)$. Further analysis of fish (e.g. fugu, medaka, and stickleback) VPAC2RA and VPAC2RB for PHIR characteristics is essential. First, to use fugu as an 


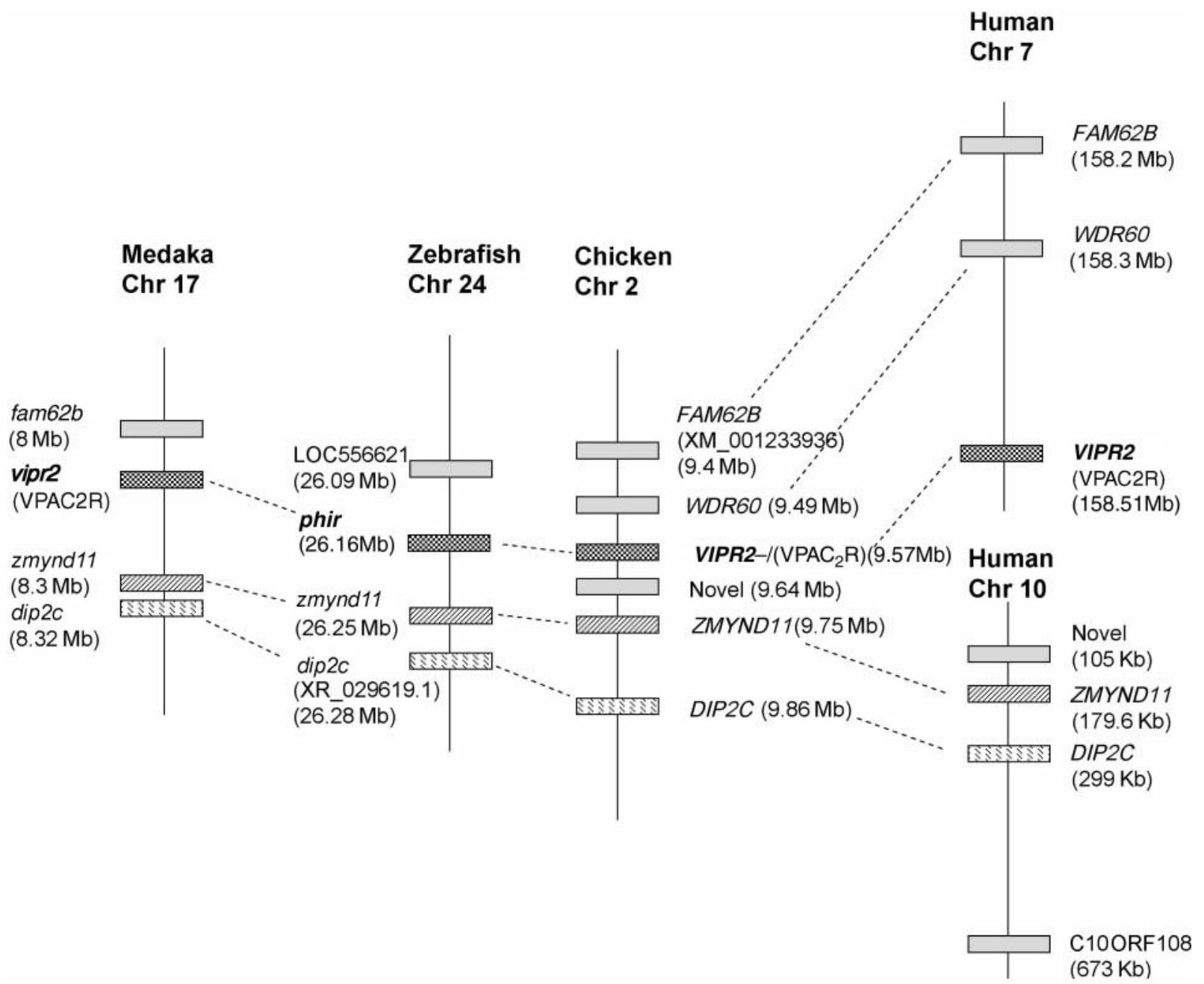

Figure 5 Chromosomal location of phir for zebrafish and VIPR2 (VPAC2R) for medaka, chicken, and human. Two linked genes (ZMYND11 and DIP2C) in the same chromosomal region as the receptors are shown to be in corresponding positions for zebrafish, medaka, and chicken. However, in the human genome, VIP2R is on chromosome 7, whereas the 'linked' genes are on chromosome 10. phir, peptide histidine-isoleucine receptor; vipr2, VIP/PACAP shared receptor, type 2.

example, its VPAC2Rs only have two glycosylation sites each (Fig. 1B). Second, fugu VPAC2Rs share relatively high sequence identity with zebrafish PHIR: 55 and $62 \%$ of VPAC2RA and VPAC2RB respectively. Third, in $\mathrm{NJ}$ and ML phylogenetic trees, fugu VPAC2RA and VPAC2RB cluster more closely with zebrafish PHIR than with chicken and human VPAC2R. However, the sequence similarity may not represent functional equivalence as addressed by Cardoso et al. (2005). Whether fugu VPAC2RA and VPAC2RB are PHI receptors will be defined by functional assays.

One hypothesis is that the tetrapod VPAC2R and the teleost PHIR shared a common origin. Support for this hypothesis includes the close structural identity between the two types of receptors (Fig. 1), the chromosomal location and linked genes (Fig. 5), and the activation of the human VPAC2R in which zebrafish PHI $\left(\mathrm{EC}_{50}=7 \cdot 4 \times 10^{-9} \mathrm{M}\right)$ is more potent than human PHM $\left(\mathrm{EC}_{50}=4.7 \times 10^{-8} \mathrm{M}\right.$; Fig. 6E). Common ancestry is also suggested in that zebrafish PHI is a potent stimulator of both the zebrafish PHIR $\left(1.8 \times 10^{-9} \mathrm{M}\right)$ and human VPAC2R $\left(7 \cdot 4 \times 10^{-9} \mathrm{M}\right.$; Fig. $6 \mathrm{E}$ and $\mathrm{H})$. However, a phylogenetic map for peptide affinity changes cannot be prepared until a number of other so-called VPAC2Rs are tested with a species-specific PHI. This is especially true for the teleost fish where two forms of VPAC2Rs have been annotated in the genome; the duplicates are presumably due to a large scale or whole genome duplication. But the duplicate VPAC2Rs in fish has not yet been cloned and their start sites are ambiguous (Fig. 1). If the duplicates are functional, it is possible that one receptor is a PHI receptor and the other has a different function, possibly as a VPAC2R. Mapping of the receptor changes using affinity values to PHI, VIP, and PACAP should help to elucidate the proposed receptor status. 


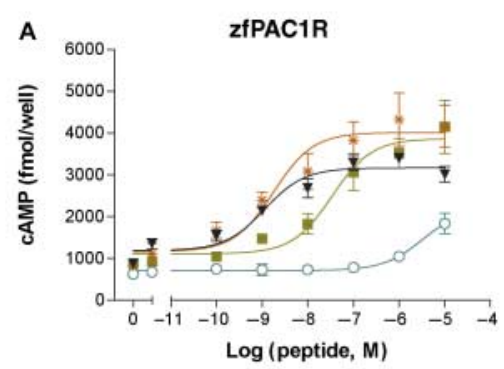

- zfPACAP 27 type1

* zfPACAP 27 typer

- zfVIP

- zfPHI

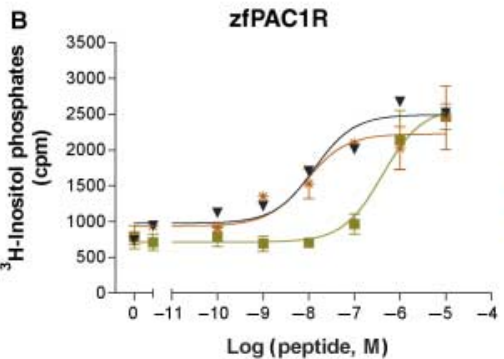

- zfPACAP 27 type 1

* zfPACAP 27 type2

- zfVIP

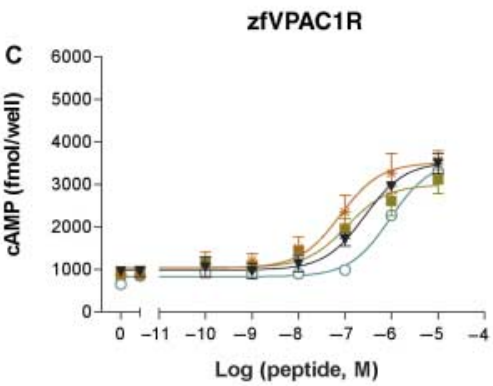

- zfPACAP 27 type1

* zfPACAP 27 type 2

- ZfVIP

- zfPHI
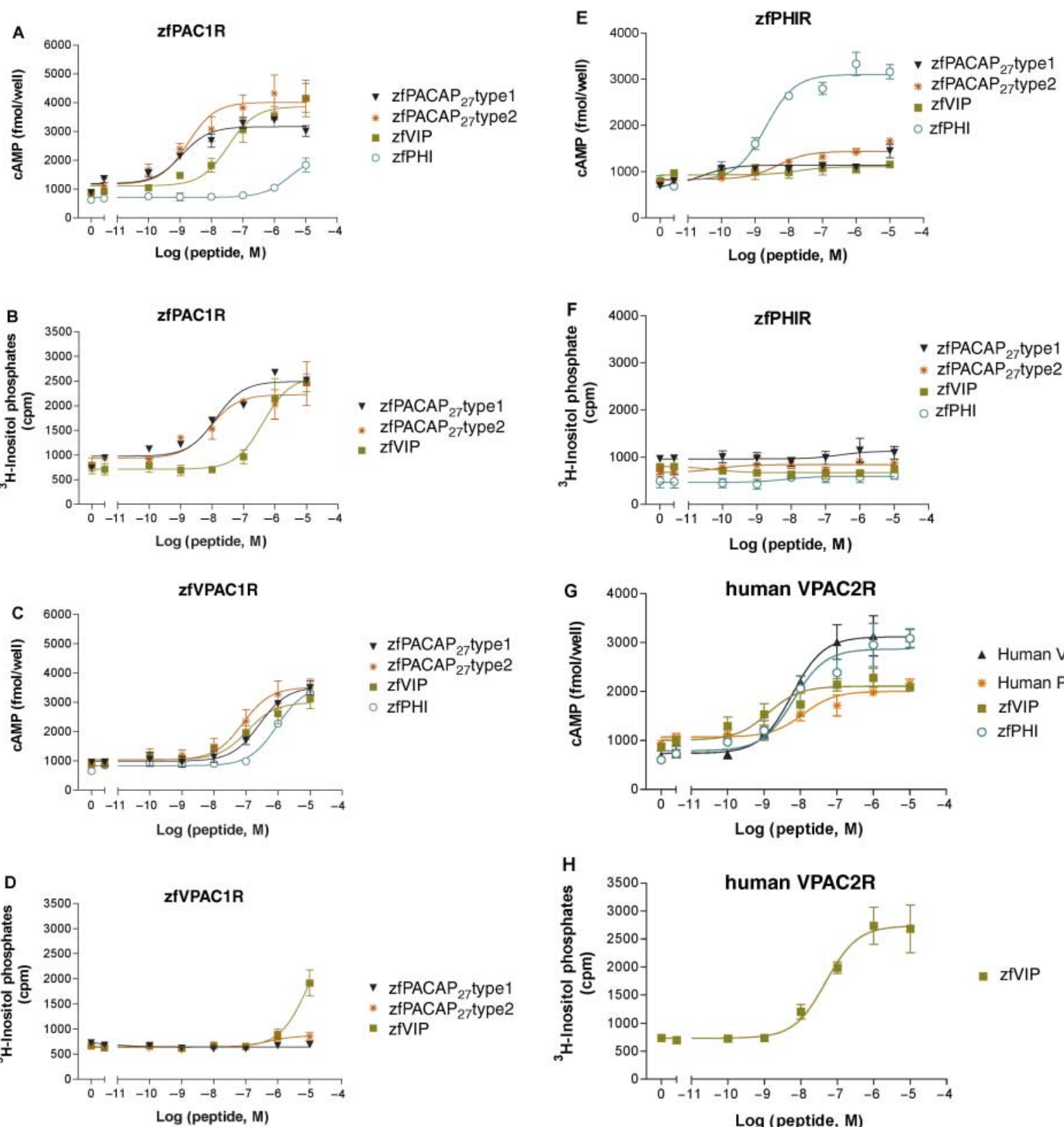

\section{A Human VIP \\ * Human PHM \\ a zfVIP \\ - zfPHI}

- zfPACAP ${ }_{27}$ type1

* zfPACAP 27 type2

- zfVIP

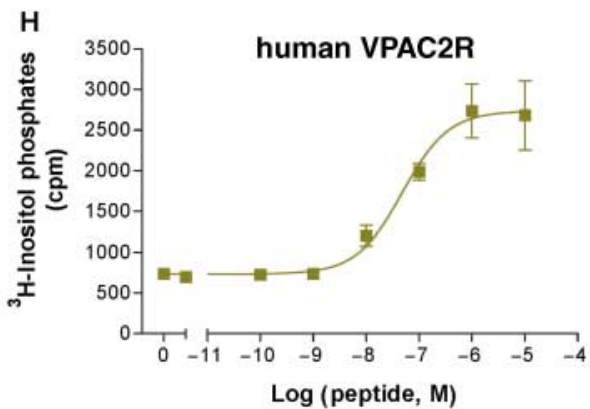

Figure 6 Accumulation of cAMP and inositol phosphate (IP) in COS7L cells transfected with different receptors and stimulated with graded concentrations of zebrafish (zf) and human peptides. (A) cAMP and (B) IP of zebrafish PAC1R; (C) cAMP and (D) IP of zebrafish VPAC1R; (E) cAMP and (F) IP of zebrafish PHIR; (G) CAMP and (H) IP of human VPAC2R. Values represent means \pm s.E.M. from a minimum of three independent experiments each in triplicate. PACAP, pituitary adenylate cyclase-activating polypeptide; PHI, peptide histidine-isoleucine; PHM, peptide histidine-methionine; VIP, vasoactive intestinal polypeptide; PAC1R, PACAP receptor; VPAC1R, VIP/PACAP shared receptor, type 1; VPAC2R, VIP/PACAP shared receptor, type 2. Full colour version of this figure available via http://dx.doi.org/10.1677/JME-08-0083

\section{Zebrafish PAC1R-short has unconventional response to VIP}

It was generally accepted that PAC1R specifically binds PACAP with a very low affinity to VIP and that only VPAC1R and VPAC2R have similar affinity to both PACAP and VIP (Vaudry et al. 2000). This is true for PAC1R-short, which is the basic form, and for the PAC1Rs that have added cassettes in the third intracellular loop. However, we show here that the PAC1R-short isoform in zebrafish has a significant dose-dependent response to PACAPs and VIP in both cAMP and IP assays. Although VIP is not as potent as the PACAP $\left(\mathrm{EC}_{50}=10^{-9} \mathrm{M}\right)$ in activating the PAC1R, the $\mathrm{EC}_{50}$ value of zebrafish VIP is a physiological 
Table $6 \mathrm{EC}_{50}$ values of receptors response to peptides

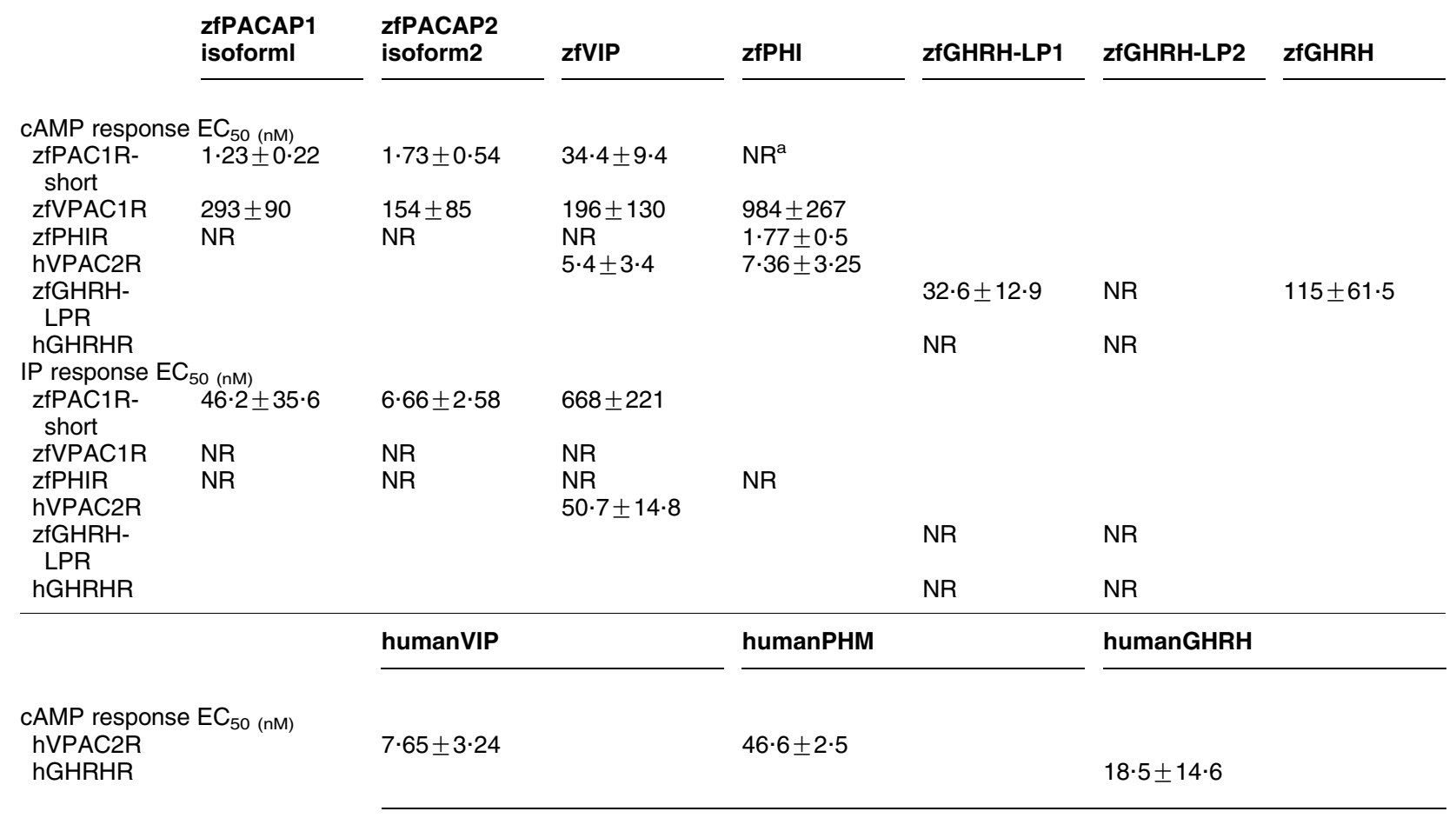

${ }^{a} \mathrm{NR}$, no response at $<10^{-6} \mathrm{M}$ peptide.

concentration $\left(\mathrm{EC}_{50}=4 \cdot 7 \times 10^{-8} \mathrm{M}\right)$ and there was no significant difference $(P<0 \cdot 05)$ between PAC1R and VPAC1R in response to VIP in the cAMP path. Support for the VIP action on the PAC1R comes from a recent paper on sea bream in which human VIP activated the sea bream PAC1R $\left(\mathrm{EC}_{50}=10^{-7} \mathrm{M}\right)$, although less than the PACAPs $\left(\mathrm{EC}_{50}=10^{-9} \mathrm{M}\right.$; Cardoso et al. 2007b). Relevant also is a report that there are at least 14 variants of the PAC1R in human neuroblastoma cells due to alternative splicing and some of these variants also respond to VIP stimulation in a dose-dependent manner in the cAMP and IP signaling pathways (Lutz et al. 2006). Likewise, human fetal brain has PAC1R variants that respond to VIP (Lutz et al. 2006). It is possible that certain variant PAC1Rs in both fish and human retained the function of responding to VIP. Moreover, human VPAC2R also responds well to zebrafish VIP with no significant difference $(P>0.05)$ compared with zebrafish PAC1R in both the cAMP and IP signaling pathways. The zebrafish VIP peptide shares $82 \%$ amino acid identity with human VIP.

\section{Zebrafish GHRH-LPR responds to both zebrafish GHRH-LPR and GHRH}

In the present study, we identified a new receptor in zebrafish, which we argue is GHRH-LPR because it has $88 \%$ sequence identity with goldfish GHRH-LPR but only $40 \%$ identity with zebrafish and human GHRHR (Lee et al. 2007). Sequence alignment with goldfish and fugu GHRH-LPRs reveals closely related structures: 13 conserved cysteines, three $N$-glycosylation sites, and RLAK for the coupling to $\mathrm{G}_{\mathrm{s}}$ and FQGBBVXXBYCFXN$\mathrm{XEXQ}$ ( $\mathrm{B}$ is a hydrophobic residue and $\mathrm{X}$ is any residue), characteristic of secretin family receptors. Also phylogenetic analysis grouped zebrafish and goldfish GHRH-LPRs along with other GHRH-LPRs with a bootstrap value of 100 . The second form of zebrafish GHRH-LPR also clustered with the same group, as it has seven transmembrane domains but it lacks 100 amino acids at the N-terminus.

Functional assays revealed that the GHRH-LP receptors in both zebrafish and goldfish are most sensitive to one of their two GHRH-LPs but both also respond to their GHRH with about threefold less potency in zebrafish and 30-fold less in goldfish (Lee et al. 2007). The activation of the zebrafish GHRH-LPR was concentration dependent in the cAMP pathway and the potency of zebrafish GHRH-LP1 and zebrafish GHRH was not significantly different $(P>0 \cdot 05)$. Similarly, Lee et al. (2007) found that the goldfish GHRHLPR (PRPR) was stimulated by one of two goldfish GHRH-LPs, and by fish GHRH. The $\mathrm{EC}_{50}$ values for fish GHRH tested in goldfish $\left(1.8 \times 10^{-7} \mathrm{M}\right)$ and zebrafish $\left(1.15 \times 10^{-7} \mathrm{M}\right)$ are nearly the same. Nonetheless, a more potent response occurs with GHRH-LP1; the $\mathrm{EC}_{50}$ 

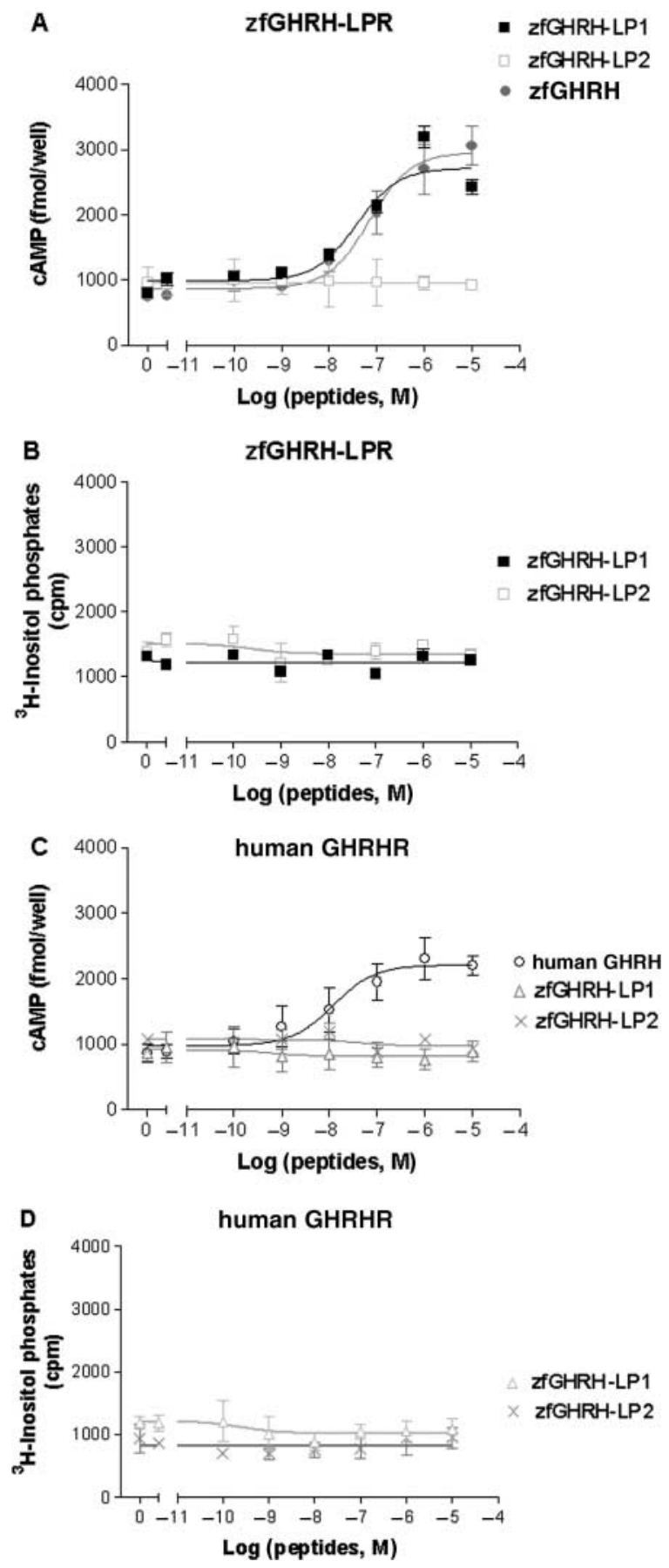

Figure 7 Functional assay using graded concentrations of zebrafish GHRH-LP1, GHRH-LP2, and GHRH on (A) CAMP and (B) IP accumulation in COS7L cells transfected with zebrafish growth hormone-releasing hormone-like peptide receptor (zebrafish GHRH-LPR); (C) CAMP and (D) IP accumulation in COS7L cells transfected with human GHRHR. Also, the latter was tested with human GHRH (cAMP). Values in cAMP assay represent means \pm S.E.M. from three independent experiments each in triplicate. Values in IP assay represent means \pm S.E.M. from at least two independent experiments each in triplicate. values are $5 \cdot 8 \times 10^{-9} \mathrm{M}$ for goldfish and $3 \cdot 41 \times 10^{-8} \mathrm{M}$ for zebrafish. Moreover, neither zebrafish nor goldfish GHRH-LPR responded to its second form of GHRH-LP. We did not detect any response of human GHRHR to either form of zebrafish GHRH-LP. We conclude that zebrafish GHRH-LPR (PRP) has at least two specific ligands: zebrafish GHRH-LP1 and zebrafish GHRH in the cAMP path. However, zebrafish GHRH receptor only responds to fish GHRH, not to goldfish GHRH-LP stimulation (Lee et al. 2007).

The homologous receptor in humans (PRPR) has been lost from the human genome (Cardoso et al. 2007 a, Lee et al. 2007). This implies that the function of GHRH-LP is distinct for fish and other non-mammals compared with mammals.

\section{Cleavage of zebrafish prepro-PHI/VIP is predicted to produce two amidated peptides or extended forms without amidation}

PHI and VIP are both transcribed from the same gene in vertebrates. PHI has an amino acid cleavage site of GKR, which implies that PHI is amidated at position 27. Another zebrafish peptide, PHM, is a C-terminal extension of PHI to a methionine at position 42 but is not amidated, as it is followed by only a KR cleavage site. VIP also has a GKR cleavage site after position 28. The zebrafish VIP C-terminus can be extended, which is similar to human VIP. Both PHI and VIP are amidated at the C-terminus but goldfish and chicken PHIs are not amidated because of the lack of a glycine residue (Talbot $e t$ al. 1995, Tse et al. 2002). Also, a VIP extension is absent in goldfish because $\mathrm{VIP}_{28}$ is directly followed by a stop codon. The primary structure of zebrafish PHI has been conserved ( $78 \%$ amino acid identity) compared with the human $\mathrm{PHM}_{27}$. In general, PHI/ PHM activates all known mammalian VIP receptors (VPACR) with moderate to weak activation (Moriarty et al. 1984, Palle et al. 1989, Lelièvre et al. 1998). However, Lelièvre et al. (1998) suggested that PHI had its own specific receptor that preferentially binds PHI over VIP. In their study, PHI had a 100fold more potent anti-proliferative action than VIP using radio-labeled $\mathrm{PHI}$ as a tracer for binding studies on neuroblastoma cells. The study also revealed that there were high-affinity receptors that selectively bound PHI over VIP, although neuroblastoma cells are known to express many alternatively spliced receptors with different binding profiles (Lutz et al. 2006) The existence of zebrafish and goldfish PHIR and the high conservation of PHI in fish and human suggest that both VIP and PHI play an important role in fish and possibly other vertebrates. 


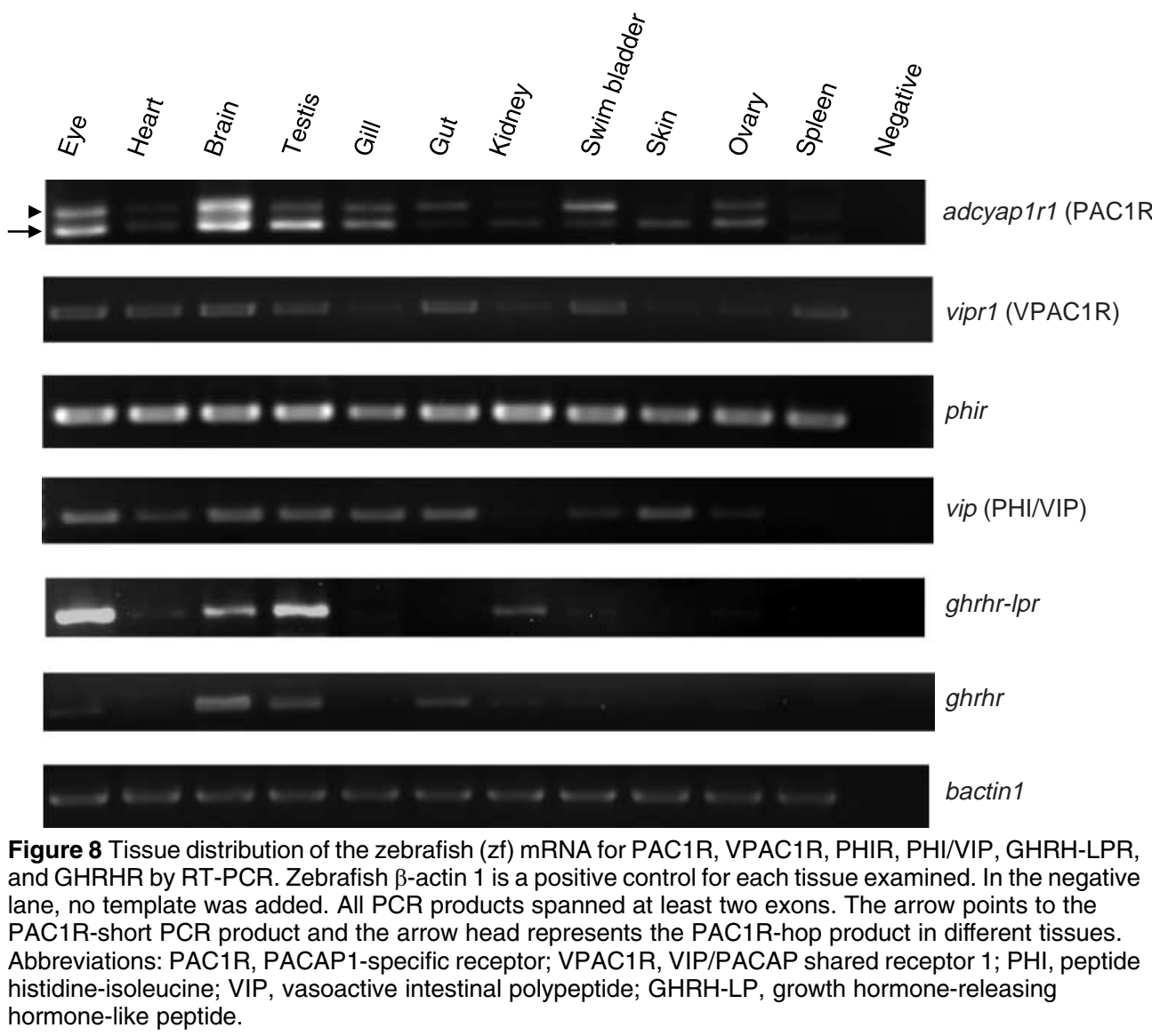

\section{Location of receptors indicates diverse target tissues for peptides}

To compare the location of zebrafish PHIR and GHRHLPR with other related peptide receptors, we performed an RT-PCR study. PHIR is one of the most widely distributed receptors and its mRNA highly expressed in all 11 tissues. The co-expression of PHIR with PHI in the eye, heart, brain, testis, gill, gut, swimbladder, skin, and ovary indicates that PHI may act in an autocrine/ paracrine manner. Goldfish PHIR expression was quantified by real-time PCR (Tse et al. 2002). High expression levels were found in the brain, heart, testis, and gut, which are similar to zebrafish.

Zebrafish PAC1R-short mRNA is expressed widely except in the spleen. This result is similar to the observation of Fradinger et al. (2005), except we found additional weak expression in the heart and swimbladder. Goldfish PAC1R-short mRNA was not detected in the spleen either but has strong expression in the heart measured by real-time PCR (Kwok et al. 2006). The PAC1R-short isoform is one of the most widely distributed receptors tested here, along with PHIR in goldfish and zebrafish. In frog and mammal, the distribution was largely investigated by RT-PCR or in situ hybridization in the brain and pituitary (Hashimoto $\mathrm{et} \mathrm{al}$. 1996, Shioda et al. 1997, Hu et al. 2000) where PAC1Rs are abundantly expressed.

By contrast, the distribution of zebrafish mRNA for GHRH-LPR and GHRHR are limited compared with other receptors studied here. This probably relates to tissue-specific functions. Zebrafish GHRH mRNA was only strongly expressed in brain, testis, and gut with weak signals in eye, kidney, swimbladder, skin, and ovary. GHRH-LPR mRNA was only detected in eye, brain, testis, and kidney with weak signals in the heart. But GHRHLPR was expressed strongly in the eye compared with GHRHR. The distribution of zebrafish GHRH-LPR is comparable with a previous study in our laboratory (Fradinger et al. 2005).

\section{Conclusions}

The newly isolated zebrafish PHIR and GHRH-LPR contribute to a better understanding of the family B receptors from an evolutionary perspective. Characterization of these receptors helps to answer some of the questions posed in the Introduction. For question 1, 
our data show that PHI does have a specific receptor although to date, it is known only for zebrafish and goldfish and not mammals. We suggest that PHIR and VPAC2R had a common ancestor, as they have high sequence conservation. Small changes in the common receptor, possibly after the fish-tetrapod split, may have transformed it to a PHIR or VPAC2R in evolution; one piece of evidence is that zebrafish VIP does not activate the zebrafish PHIR but does activate the human VPAC2R. To confirm the receptor change, many other PHI/VPAC2Rs in other species need to be tested with PHI, VIP, and PACAP to establish an affinity profile. In addition, the change in peptide structure during evolution may also be important, as zebrafish PHI not only activates its receptor but is more potent than human PHM when tested with the human VPAC2R in our experiments. Questions 2 and 3 about the lack of a specific VIP receptor suggest the possibility that the original PAC1R and VPAC1R responded to both PACAP and VIP, as in zebrafish. During evolution, PAC1R may have lost its sensitivity to VIP. However, humans do have a PAC1R variant that responds to VIP and PACAP; the variant was identified not only in neuroblastoma cells but in fetal brain (Lutz et al. 2006). PACAP and VIP may have shared a common origin, as the peptides have a high sequence identity of $68 \%$ for human PACAP versus hVIP and $78-81 \%$ for zebrafish PACAP1 or PACAP2 versus zebrafish VIP. In question 4 about the PRPR (GHRH-LPR), the ligand in all vertebrates regardless of its name is encoded by the PACAP gene. The structural similarity of PRP (and PHM) to GHRH was noted in an early paper describing the gene that encodes PRP/ PACAP (Ohkubo et al. 1992). In human, the PRP sequence has a higher identity with $\mathrm{GHRH}_{27}(52 \%)$ than with PACAP $_{27}(22 \%)$. In zebrafish, the same pattern occurs in which zebrafish GHRH-LP1 is closer in structure to zebrafish $\mathrm{GHRH}_{27}(59 \%)$ than with zebrafish PACAP $_{27}$ (37\%). The structural relationship of the upstream-encoded peptide in each of three genes (for PRP/PACAP, PHM/VIP and GHRH/C-terminal peptide) and also the downstream peptides of PACAP and VIP (see above) suggest the genes resulted from at least two duplications in evolution, which must have occurred before the fish-tetrapod split as teleosts have the same three genes as mammals. A substitution in the first amino acid of mammalian PRPs and the apparent loss of their receptors (Cardoso et al. 2007a, Lee et al. 2007) may have resulted in a hormone that no longer activates cAMP (Okazaki et al. 1992). Although the function of GHRH-LPs is not clear, the name should remain, as these peptides are structurally closer to GHRH than to PACAP. Hence, PRP is less a PACAP-related peptide than a PACAP gene-associated peptide. The answer to question 5 as to whether zebrafish is a good model for endocrine studies is shown in Table 1. These fish have a full complement of secretin superfamily hormones except for secretin and its receptor, although the status of duplicate receptors is at an early state of study. The zebrafish superfamily has matching receptors for its known hormones including PHIR and GHRH-LPR (PRPR), unknown in humans to date.

\section{Declaration of interest}

The authors declare that there is no conflict of interest that would prejudice the impartiality of this scientific work.

\section{Funding}

Thanks are due to the Candian Natural Sciences and Engineering Research Council (NSERC) for funding.

\section{Acknowledgements}

We thank Dr Javier Tello for teaching the receptor assay techniques and Drs John Taylor and Gustavo Ybazeta for help with the maximumlikelihood analysis.

\section{References}

Adams BA, Tello JA, Erchegyi J, Warby C, Hong DJ, Akinsanya KO, Mackie GO, Vale W, Rivier JE \& Sherwood NM 2003 Six novel gonadotropin-releasing hormones are encoded as triplets on each of two genes in the protochordate, Ciona intestinalis. Endocrinology 144 1907-1919.

Cardoso JC, Power DM, Elgar G \& Clark MS 2004 Duplicated receptors for VIP and PACAP (VPAC1R and PAC1R) in a teleost fish, Fugu rubripes. Journal of Molecular Endocrinology 33 411-428.

Cardoso JC, Clark MS, Viera FA, Bridge PD, Gilles A \& Power DM 2005 The secretin G-protein-coupled receptor family: teleost receptors. Journal of Molecular Endocrinology 34 753-765.

Cardoso JCR, Vieira FA, Gomes AS \& Power DM $2007 a$ PACAP, VIP and their receptors in the metazoa: insights about the origin and evolution of the ligand-receptor pair. Peptides 28 1902-1919.

Cardoso JCR, de Vet ECJM, Louro B, Elgar G, Clark MS \& Power DM $2007 b$ Persistence of duplicated PAC1 receptors in the teleost, Sparus auratus. BMC Evolutionary Biology 7221.

Chan KW, Yu KL, Rivier J \& Chow BK 1998 Identification and characterization of a receptor from goldfish specific for a teleost growth hormone-releasing hormone-like peptide. Neuroendocrinology 68 44-56.

Chengalvala M, Kostek B \& Frail DE 1999 A multi-well filtration assay for quantitation of inositol phosphates in biological samples. Journal of Biochemical and Biophysical Methods 38 163-170.

Chow BK, Yuen TT \& Chan KW 1997 Molecular evolution of vertebrate VIP receptors and functional characterization of a VIP receptor from goldfish Carassius auratus. General and Comparative Endocrinology 105 176-185.

Chow BK, Pang RT \& Ng SS 2003 Molecular evolution of PACAP precursor and PACAP receptors. In Pituitary Adenylate CyclaseActivating Polypeptide, pp 23-47. Eds H Vaudry \& A Arimura. Norwell, MA: Kluwer Academic Publishers.

Couvineau A, Gaudin P, Maoret JJ, Rouyer-Fessard C, Nicole P \& Laburthe M 1995 Highly conserved aspartate 68, tryptophane 73 
and glycine 109 in the N-terminal extracellular domain of the human VIP receptor are essential for its ability to bind VIP. Biochemical and Biophysical Research Communications 206 246-252.

Couvineau A, Fabre C, Gaudin P, Maoret JJ \& Laburthe M 1996 Mutagenesis of $\mathrm{N}$-glycosylation sites in the human vasoactive intestinal peptide 1 receptor. Evidence that asparagine 58 or 69 is crucial for correct delivery of the receptor to plasma membrane. Biochemistry 35 1745-1752.

Foord SM, Bonner TI, Neubig RR, Rosser EM, Pin JP, Davenport AP, Spedding M \& Harmar AJ 2005 International Union of Pharmacology. XLVI. G protein-coupled receptor list. Pharmacological Reviews 57 279-288.

Fradinger EA \& Sherwood NM 2000 Characterization of the gene encoding both growth hormone-releasing hormone (GRF) and pituitary adenylate cyclase-activating polypeptide (PACAP) in the zebrafish. Molecular and Cellular Endocrinology 165 211-219.

Fradinger EA, Tello JA, Rivier JE \& Sherwood NM 2005 Characterization of four receptor cDNAs: PAC1, VPAC1, a novel PAC1 and a partial GHRH in zebrafish. Molecular and Cellular Endocrinology 231 $49-63$.

Guindon S \& Gascuel O 2003 A simple, fast, and accurate algorithm to estimate large phylogenies by maximum likelihood. Systematic Biology 52 696-704.

Guindon S, Lethiec F, Duroux P \& Gascuel O 2005 PHYML Online - a web server for fast maximum likelihood-based phylogenetic inference. Nucleic Acids Research 33 W557-W559.

Hashimoto H, Nogi H, Mori K, Ohishi H, Shigemoto R, Yamamoto K, Matsuda T, Mizuno N, Nagata S \& Baba A 1996 Distribution of the mRNA for a pituitary adenylate cyclase-activating polypeptide receptor in the rat brain: an in situ hybridization study. Journal of Comparative Neurology 371 567-577.

Hoo RL, Alexandre D, Chan SM, AnouarY, Pang RT, Vaudry H \& Chow BK 2001 Structural and functional identification of the pituitary adenylate cyclase-activating polypeptide receptor VPAC2 from the frog Rana tigrina rugulosa. Journal of Molecular Endocrinology 27 229-238.

Hu Z, Lelievre V, Tam J, Cheng JW, Fuenzalida G, Zhou X \& Waschek JA 2000 Molecular cloning of growth hormone-releasing hormone/pituitary adenylyl cyclase-activating polypeptide in the frog Xenopus laevis: brain distribution and regulation after castration. Endocrinology 141 3366-3376.

Kumar S, Tamura K \& Nei M 2004 MEGA3: Integrated software for molecular evolutionary genetics analysis and sequence alignment. Briefings in Bioinformatics 5 150-163.

Kwok YY, Chu JY, Vaudry H, Yon L, Anouar Y \& Chow BK 2006 Cloning and characterization of a PAC1 receptor hop-1 splice variant in goldfish (Carassius auratus). General and Comparative Endocrinology 145 188-196.

Laburthe M, Couvineau A \& Nicole P 2003 Molecular pharmacology and structure-function analysis of PACAP/VIP receptors. In Pituitary Adenylate Cyclase-Activating Polypeptide, pp 69-93. Eds H Vaudry \& A Arimura. Norwell, MA: Kluwer Academic Publishers.

Lee LT, Siu FK, Tam JK, Lau IT, Wong AO, Lin MC, Vaudry H \& Chow BK 2007 Discovery of growth hormone - releasing hormones and receptors in nonmammalian vertebrates. PNAS 104 2133-2138.

Lelièvre V, Pineau N, Du J, Wen CH, Nguyen T, Janet T, Muller JM \& Wascheck JA 1998 Differential effects of peptide histidine isoleucine (PHI) and related peptides on stimulation and suppression of neuroblastoma cell proliferation. Journal of Biological Chemistry 273 19685-19690.

Lok S, Kuijper JL, Jelinek LJ, Kramer JM, Whitmore TE, Sprecher CA, Mathewes S, Grant FJ, Biggs SH, Rosenberg GB et al. 1994 The human glucagon receptor encoding gene: structure, cDNA sequence and chromosomal localization. Gene 40 203-209.

Lutz EM, Ronaldson E, Shaw P, Johnson MS, Holland PJ \& Mitchell R 2006 Characterization of novel splice variants of the PAC1 receptor in human neuroblastoma cells: consequences for signaling by VIP and PACAP. Molecular and Cellular Neurosciences 31 193-209.

McGonnell IM \& Fowkes RC 2006 Fishing for gene function endocrine modelling in the zebrafish. Journal of Endocrinology 189 $425-439$.

Miyata A, Arimura A, Dahl RR, Minamino N, Uehara A, Jiang L, Culler MD \& Coy DH 1989 Isolation of a novel 38 residue-hypothalamic polypeptide which stimulates adenylate cyclase in pituitary cells. Biochemical and Biophysical Research Communications 164 567-574.

Miyata A, Jiang L, Dahl RD, Kitada C, Kubo K, Fujino M, Minamino N \& Arimura A 1990 Isolation of a neuropeptide corresponding to the N-terminal 27 residues of the pituitary adenylate cyclase activating polypeptide with 38 residues (PACAP 38). Biochemical and Biophysical Research Communications 170 643-648.

Moriarty KJ, Hegarty JE, Tatemoto K, Mutt V, Christofides ND, Bloom SR \& Wood JR 1984 Effect of peptide histidine isoleucine on water and electrolyte transport in the human jejunum. Gut $\mathbf{2 5}$ 624-628.

Ohkubo S, Kimura C, Ogi K, Okazaki K, Hosoya M, Onda H, Miyata A, Arimura A \& Fujino M 1992 Primary structure and characterization of the precursor to human pituitary adenylate cyclase activating polypeptide. DNA and Cell Biology 11 21-30.

Okazaki K, Kimura C, Kosaka T, Watanabe T, Ohkubo S, Ogi K, Kitada C, Onda H \& Fujino M 1992 Expression of human pituitary adenylate cyclase activating polypeptide (PACAP) cDNA in CHO cells and characterization of the products. FEBS Letters 298 49-56.

Palle C, Ottesen B, Jorggensen J \& Fahrenkrug J 1989 Peptide histidine methionine and vasoactive intestinal peptide: occurrence and relaxant effect in the human female reproductive tract. Biology of Reproduction 41 1103-1111.

Parker DB, Power ME, Swanson P, Rivier J \& Sherwood NM 1997 Exon skipping in the gene encoding pituitary adenylate cyclase-activating polypeptide in salmon alters the expression of two hormones that stimulate growth hormone release. Endocrinology 138 414-423.

Sherwood NM, Krueckl SL \& McRory JE 2000 The origin and function of the pituitary adenylate cyclase-activating polypeptide (PACAP)/glucagon superfamily. Endocrine Reviews 21 619-670.

Shioda S, Yada T, Nakajo S, Nakaya K, Nakai Y \& Arimura A 1997 Pituitary adenylate cyclase-activating polypeptide (PACAP): a novel regulator of vasopressin-containing neurons. Brain Research 765 81-90.

Solano RM, Langer I, Perret J, Vertongen P, Juarranz MG, Robberecht P \& Waelbroeck M 2001 Two basic residues of the h-VPAC1 receptor second transmembrane helix are essential for ligand binding and signal transduction. Journal of Biological Chemistry 276 1084-1088.

Talbot RT, Dunn IC, Wilson PW, Sang HM \& Sharp PJ 1995 Evidence for alternative splicing of the chicken vasoactive intestinal polypeptide gene transcript. Journal of Molecular Endocrinology 15 81-91.

Tam JKV, Lee LTO \& Chow BKC 2007 PACAP-related peptide (PRP)Molecular evolution and potential functions. Peptides 28 1920-1929.

Tello JA, Rivier JE \& Sherwood NM 2005 Tunicate gonadotropinreleasing hormone $(\mathrm{GnRH})$ peptides selectively activate Ciona intestinalis GnRH receptors and the green monkey type II GnRH receptor. Endocrinology 146 4061-4073.

Thompson JD, Higgins DG \& Gibson TJ 1994 CLUSTAL W: improving the sensitivity of progressive multiple sequence alignment through sequence weighting, positions-specific gap penalties and weight matrix choice. Nucleic Acids Research 22 4673-4680.

Tse DL, Pang RT, Wong AO, Chan SM, Vaudry H \& Chow BK 2002 Identification of a potential receptor for both peptide histidine isoleucine and peptide histidine valine. Endocrinology 143 1327-1336.

Ulloa-Aguirre A \& Conn PM 2000 G protein-coupled receptors and G proteins. In Principles of Molecular Regulation, pp 3-25. Eds PM Conn \& R Means. Totowa, NJ: Humana Press.

Vaudry D, Gonzalez BJ, Basille M, Yon L, Fournier A \& Vaudry H 2000 Pituitary adenylate cyclase-activating polypeptide and its receptors: from structure to functions. Pharmacological Reviews 52 269-324. 
Wang Y, Wong AO \& Ge W 2003 Cloning, regulation of messenger ribonucleic acid expression, and function of a new isoform of pituitary adenylate cyclase-activating polypeptide in the zebrafish ovary. Endocrinology 144 4799-4810.

Wang Y, Li J, Wang CY, Kwok AH \& Leung FC 2007 Identification of the endogenous ligands for chicken growth hormone-releasing hormone (GHRH) receptor: evidence for a separate gene encoding GHRH in submammalian vertebrates. Endocrinology 148 2405-2416.

Wong AOL, Leung MY, Shea WLC, Tse LY, Chang JP \& Chow BKC 1998 Hypophysiotropic action of pituitary adenylate cyclase-activating polypeptide (PACAP) in the goldfish: immunohistochemical demonstration of PACAP in the pituitary, PACAP stimulation of growth hormone release from pituitary cells, and molecular cloning of pituitary type I PACAP receptor. Endocrinology 139 3465-3479.

Received in final form 15 August 2008 Accepted 29 August 2008

Made available online as an Accepted Preprint 29 August 2008 